\title{
An Efficient Finite Element Formulation of Dynamics for a Flexible Robot with Different Type of Joints
}

Chu A My ${ }^{* 1}$, Duong X Bien ${ }^{1}$, Chi Hieu Le ${ }^{2}$, Michael Packianather ${ }^{3}$

${ }^{1}$ Department of Special Robotics and Mechatronics, Le Quy Don Technical University, 236 Hoang Quoc Viet, Hanoi, Vietnam.

${ }^{2}$ Faculty of Engineering and Science, University of Greenwich, Central Avenue, Chatham Maritime, Kent ME4 4TB, United Kingdom.

${ }^{3}$ School of Engineering, Cardiff University, Queen's Buildings, The Parade, Cardiff CF24 3AA, United Kingdom.

\section{Abstract}

If two adjacent links of a flexible robot are connected via a revolute joint or a fixed prismatic joint, the relative motion of the next link will depend on both the joint motion and the elastic displacement of the distal end of the previous link. However, if the two adjacent links are connected via a sliding prismatic joint, the relative motion of the next link will depend additionally on the elastic deformation distributed along the previous link. Therefore, formulation of the motion equations for a multi-link flexible robot consisting of the revolute joints, the fixed prismatic joints and the sliding prismatic joints is challenging. In this study, the finite element kinematic and dynamic formulation was successfully developed and validated for the flexible robot, in which a transformation matrix is proposed to describe the kinematics of both the joint motion and the link deformation. Additionally, a new recursive formulation of the dynamic equations is introduced. As compared with the previous methods, the time complexity of the formulation is reduced by $\mathrm{O}(2 \eta)$, where $\eta$ is the number of finite elements on all links. The numerical examples and experiments were implemented to validate the proposed kinematic and dynamic modelling method.

\section{Keywords}

Flexible robots, flexible robotic arms, kinematics, dynamics, finite elements method. 


\section{Nomenclature}

\begin{tabular}{|c|c|}
\hline$R$ & Revolute joint \\
\hline$P_{a}$ & Sliding prismatic joint \\
\hline$P_{b}$ & Fixed prismatic joint \\
\hline$n$ & Total number of links \\
\hline$i$ & Index of a link $(i=1 \div n)$ \\
\hline$l_{i}$ & Length of a link $i$ \\
\hline$n_{i}$ & Total number of elements of a link $i$ \\
\hline$j$ & Index of element of a link $i\left(j=1 \div n_{i}\right)$ \\
\hline$l_{i e}$ & Length of elements of a link $i$ \\
\hline$\gamma_{i}$ & Angle between a link $i-1$ and a link $i$ \\
\hline$a_{i}$ & Rear part of a link $i$ \\
\hline$d_{i}$ & Variable of a prismatic joint $i$ connecting a link $i-1$ and a link $i$ \\
\hline$\theta_{i}$ & Variable of a revolute joint $i$ connecting a link $i-1$ and a link $i$ \\
\hline$O_{i} \equiv\left(O_{i} X_{i} Y_{i} Z_{i}\right)$ & Local coordinate system attached to a link $i$ \\
\hline$O_{0} \equiv\left(O_{0} X_{0} Y_{0} Z_{0}\right)$ & Reference coordinate system fixed to the base (link 0). \\
\hline$u_{(i)(2 j+1)}$ & $\begin{array}{l}\text { Flexural displacement at the common junction of elements } j \text { and } j+1 \text { of } \\
\text { a link } i\end{array}$ \\
\hline$u_{(i)(2 j+2)}$ & Flexural slope at the common junction of elements $j$ and $j+1$ of a link $i$ \\
\hline$u_{(i-1)(2 k+1)}$ & $\begin{array}{l}\text { Flexural displacement at the distal node of a sliding element } k \text { of a link } \\
i-1 \text { currently through the sliding prismatic joint } i\left(P_{a}\right)\end{array}$ \\
\hline$u_{(i-1)(2 k+2)}$ & $\begin{array}{l}\text { Flexural slope at the distal node of a sliding element } k \text { of a link } i-1 \\
\text { currently through the joint } i\left(P_{a}\right)\end{array}$ \\
\hline$u_{(i-1)\left(2 n_{i-1}+1\right)}$ & Flexural displacement at the distal end of a link $i-1$ \\
\hline$u_{(i-1)\left(2 n_{i-1}+2\right)}$ & Flexural slope at the distal end of a link $i-1$ \\
\hline$u_{(i-1) f}$ & $\begin{array}{l}\text { Flexural displacement at the nominal articulation point between a link } i-1 \\
\text { and a link } i \text {. }\end{array}$ \\
\hline$u_{(i-1) s}$ & $\begin{array}{l}\text { Flexural slope at the nominal articulation point between a link } i-1 \text { and a } \\
\text { link } i \text {. }\end{array}$ \\
\hline$x$ & A point on the element $j$ of a link $i$ \\
\hline$\phi_{m}(x), m=1 \div 4$ & Shape functions \\
\hline $\mathbf{S}$ & Vector of shape functions \\
\hline $\mathbf{S}_{i j}$ & Elemental vector of shape functions \\
\hline$w_{i j}(x)$ & Flexural displacement at the point $x$ on the element $j$ of a link $i$ \\
\hline $\mathbf{r}_{i j}(x)$ & Vector from $O_{i}$ to the point $x$ on the element $j$ of a link $i$ \\
\hline $\mathbf{r}_{0 i j}(x)$ & Vector from $O_{0}$ to the point $x$ on the element $j$ of a link $i$ \\
\hline $\boldsymbol{\Theta}_{(i-1) i}$ & $\begin{array}{l}\text { Transformation matrix describing the motion of a flexible link } i \text { relative to } \\
\text { a flexible link } i-1\end{array}$ \\
\hline
\end{tabular}




\begin{tabular}{|l|l|}
\hline $\mathbf{H}_{(i-1) i}$ & $\begin{array}{l}\text { Transformation matrix describing the motion of a rigid link } i \text { relative to a } \\
\text { rigid link } i-1\end{array}$ \\
\hline$m_{i}$ & Uniform density (mass per meter) of a link $i$ \\
\hline$E$ & Young's modulus \\
\hline$I_{i}$ & Moment of inertia of a link $i$ \\
\hline $\mathbf{M}$ & Global mass matrix of the system \\
\hline $\mathbf{M}_{i j}$ & Elemental mass matrix for the element $j$ of a link $i$ \\
\hline $\mathbf{K}$ & Global stiffness matrix of the system \\
\hline $\mathbf{K}_{i j}$ & Elemental stiffness matrix for the element $j$ of a link $i$ \\
\hline$p_{i}$ & Variable of a joint $i$ \\
\hline $\mathbf{p}_{i j}$ & Vector of elastic displacements and slopes of the element $j$ of a link $i$ \\
\hline $\mathbf{K}_{i}$ & Vector of elastic displacements and slopes of a link $i$ \\
\hline $\mathbf{q}$ & Vector of generalized coordinates of the system \\
\hline $\mathbf{F}$ & Vector of applied torques/forces of the system \\
\hline
\end{tabular}




\section{Introduction}

Industrial robots can be categorized as the rigid or flexible ones. The traditional rigid robots have been widely used in various industries. Today, there has been an emerging need to develop the flexible robots to meet well more and more sophisticated requirements, especially in manufacturing industries. The flexible robots have outstanding advantages over the traditional rigid ones, with a lower overall mass, smaller actuators, lower energy consumptions, and a greater payload-to-manipulatorweight ratio, taking the advantages of the latest advancements in robotics, design and manufacturing, as well as the material engineering. The control algorithms of rigid robots are insufficient when they come to flexible ones; and the mathematical modeling process for the flexible robots is much more complex, especially when working on the nonlinear control issues, and the dynamic modeling and analysis of multi-link flexible robots. Moreover, formulations of the motion equations for a multi-link flexible robot consisting of the revolute joints, the fixed prismatic joints and the sliding prismatic joints are always challenging.

Fig. 1 shows the revolute joint (Fig. 1a), the fixed prismatic joint (Fig. 1b) and the sliding prismatic joint (Fig. 1c) that are usually used in robot architectures. The fixed prismatic joint implies that it is fixed to the previous link $i-1$, the current link $i$ slides through the joint, and the length of the current link $i$ varies with respect to time. In contrast, the sliding prismatic joint is fixed to the current link $i$, both the joint and the link translate relatively to the previous link $i-1$, and the length of the previous link $i-1$ varies.

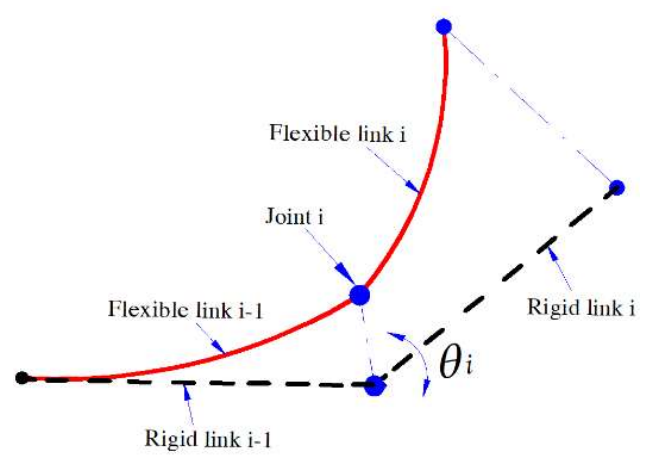

a) A revolute joint

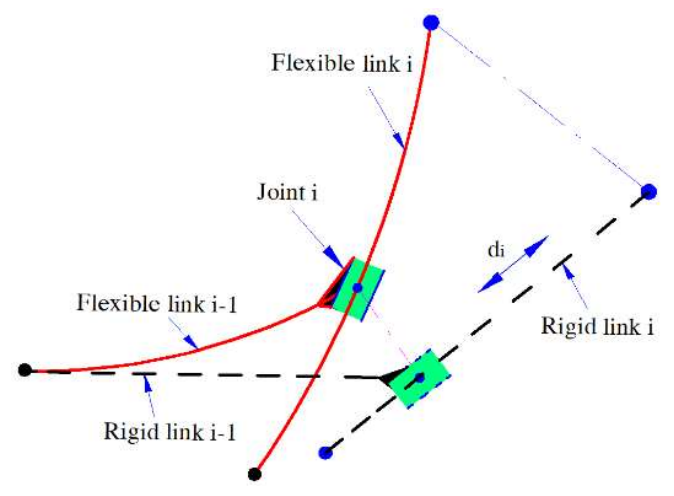

b) A fixed prismatic joint

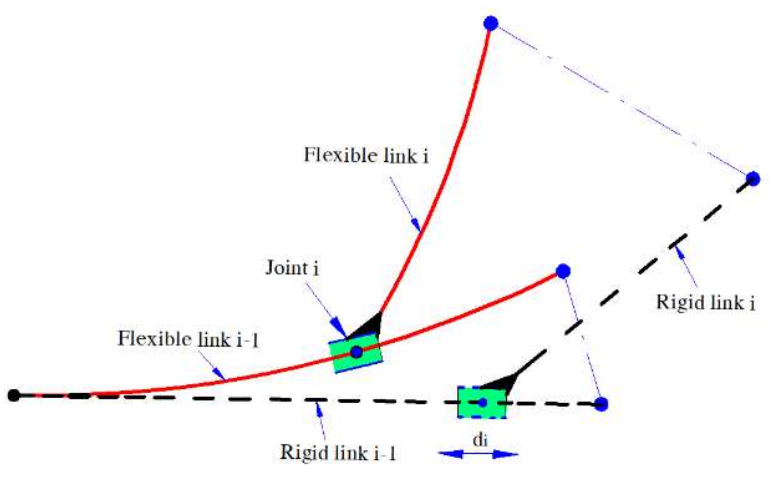

c) A sliding prismatic joint

Fig.1. Three types of joints for the multi-link flexible robots 
Generally, the kinematics of a flexible link $i$ depends much on which kind of joints connecting the link $i$ with the previous link $i-1$. For the case in which the two links are connected by a revolute or a fixed prismatic joint (Fig. 1a, 1b), the relative motion of the link $i$ depends on the motion of the joint $i$ and the elastic deformation at the distal end of the previous link $i-1$. Nevertheless, in the case of sliding prismatic joint (Fig.1c), the motion of the link $i$ does not depend on the elastic deformation at the distal end of the link $i-1$, it depends on the elastic deformation of the sliding element on the link $i-1$. This element varies along the length of the link $i-1$, with respect to time. For the cases in which the revolute joint and the sliding prismatic joint (Fig.1a, 1c), it is usually assumed that the elastic displacements at the first node of the first element on the link $i$ equal to zero. However, for the case in which the fixed prismatic joint (Fig. 1b), the element of zero elastic deformation is the sliding element of the link $i$ through the fixed prismatic joint. Obviously, the elasticity effects of links associated with the use of the three joint types should be taken into accounts when working on the kinematic and dynamic modeling for a general flexible robot that consists of all three joint types.

Decades ago, the dynamic modeling and analysis of multi-link flexible robots have been well documented (Korayem et al., 2014; Raouf et al., 2017; Augustynek and Adamiec-Wójcik, 2014; Book, 1984; Naganathan \& Soni, 1987; Jonker, 1990; Lin and Lewis, 1994; Usoro, 1986; De Luca and Siciliano, 1991; Amirouche and Xie, 1993; Chen, 2001; Subudhi and Morris, 2002; Khadem and Pirmohammadi, 2003; Zhang, 2009; Korayem et al., 2012). There have also been attempts to generalize the kinematic and dynamic modeling problems for the multi-link flexible robots, and most of these investigations focus on the multi-link flexible robots consisting of all revolute joints. There were also studies considering the use of the prismatic joints for the flexible robots; however, these studies mainly focused on the robot with one or two links only. Wang and Wei (1987) and Al-Bedoor \& Khulief (1997) investigated the dynamics of a sliding flexible link through a fixed prismatic joint. Ju et al. (2016), Yuh and Young (1991) and Pan et al. (1990) developed the modeling for a two-link flexible robot with a fixed prismatic joint. Korayem et al. (2014) addressed the dynamic modelling of a multilink flexible robot, of which each link rotates and reciprocates through a fixed prismatic joint. Wang \& Mills (2016) studied a parallel robot that has the flexible links with the sliding prismatic joints. Khadem \& Pirmohammadi (2003) formulated the dynamic equations for a multi-link flexible robot consisting of the sliding prismatic joints as well.

Although the dynamic modelling of the flexible robot that consists of the sliding prismatic joints (Fig. 1c) has been considered in the researches by Khadem \& Pirmohammadi (2003), Ju et al. (2016), and Wang \& Mills (2016), the flexible deformation of the sliding link, along which the prismatic joint slides, has been overlooked. In particular, little attention has been paid to the kinematic and dynamic modelling of the multi-link flexible robots that have simultaneously the revolute joints, the fixed prismatic joints and the sliding prismatic joints. Notice that combinations of different types of joints provide the flexibilities in design and additional functions for the multi-link robots, to help meeting well the additional technical requirements and expanding the scope of applications for the multi-link robots.

Basically, the flexible robots are the continuous dynamical systems which are often characterized by an infinite number of degrees of freedom and are governed by the nonlinear coupled differential equations; and the exact solution of such systems is not practical. In order to formulate the dynamic equations, the continuous dynamical systems are usually discretized by using two main methods, including (i) the Assumed Modes Method (AMM) and (ii) Finite Elements Method (FEM). Theodore \& Ghosal (1995) and Dwivedy \& Eberhard (2006) showed that the main drawback of AMM is the difficulty in finding the modes for links with the non-regular cross sections and the multi-link robots, and FEM normally requires fewer computations; therefore, FEM lends itself ideally suited for the modeling of multi-links flexible robots. Practically, FEM has been utilized by many authors for the dynamic formulation of flexible robots (Du et al., 1996; Płosa \& Wojciech, 2000; Jonker, 1990; Naganathan \& Soni, 1987; Tokhi et al., 2001; Al-Bedoor, \& Almusallam, 2000; Mahto, 2014; Karagulle et al., 2015, 2017; Al-Bedoor \& Khulief, 1997; Pan et al., 1990; Wang \& Mills, 2006; Usoro, 1990). In this approach, each link of a robot is divided into the finite beam elements of the same length. The kinetic energy and the potential energy of each element are calculated to formulate the elemental 
mass and stiffness matrices correspondingly. Unfortunately, the elemental mass and stiffness matrix are usually calculated in terms of the nodal coordinates. With respect to the order of entries in some global vectors of the generalized coordinates, the index of the last four rows and the last four columns of each elemental matrix is different from that of the other elemental matrices. Moreover, the elemental matrices corresponding to elements on different links have different sizes. Therefore, constructions of the global mass and stiffness matrices of governing equations usually require the complex computational procedures and transformations for assembling the elemental mass and stiffness matrices respectively. Furthermore, in the case that the flexible robot consists of the sliding and fixed prismatic joints, a construction of the global mass and stiffness matrices is even more complex since the boundary conditions vary with respect to time.

The above raised critical issues and problems lead to the motivations of developing a new kinematic and dynamic formulation for the multi-link flexible robots. In this paper, a new kinematic modelling method for a planar n-link flexible robot that consists of the revolute, fixed prismatic and sliding prismatic joints is presented and discussed. A new homogeneous transformation matrix is addressed to describe the kinematics of both the joint motion and the link deformation. Since the proposed matrix is given in a general form, it is therefore applicable to modelling of any planar flexible robots. In addition, a new recursive formulation of dynamics for the flexible robots is also presented. Based on the finite element - Lagrangian approach, all the elemental mass and stiffness matrices are transformed and expressed explicitly and globally with respect to the entire generalized coordinates. Consequently, the global mass and stiffness matrices are formulated by summing over all the elemental mass and stiffness matrices of the same size respectively. By using the symbolic computing syntaxes available in the symbolic computing environments, the formulation can be implemented in a simplified and efficient manner. The proposed method in this study is more efficient and useful, especially when compared to the common methods which use the complex schemes for assembling the global mass and stiffness matrices. In order to present validations of the newly developed and proposed dynamic modelling method for multi-link flexible robots, the numerical examples and experiments are implemented.

The rest of the paper is organized as follows. Section 2 presents a brief literature review, especially the related work as foundations for the study. Sections 3 and 4 present the kinematics, dynamics and numerical examples of the dynamic analysis of a multi-link flexible robot respectively. Section 5 presents the experiments for validation of the forward dynamic analysis results based on the proposed dynamic modelling method. Finally, the summary and conclusions are presented in Section 6.

\section{A brief literature review and related work}

In recent decades, a great deal of work has been devoted to the dynamic modeling and analysis of flexible robots. The comprehensive literature reviews of the flexible robot dynamics and control were presented by Theodore \& Ghosa (1995), Dwivedy \& Eberhard (2006), Benosman \& Le Vey (2004), Rahimi \& Nazemizadeh (2014), Kiang et al. (2015), Sayahkarajy et al. (2016), Lochan \& Subudhi (2016), and Alandoli et al. (2016). Table 1 presents the summary of the related work and key references about the dynamic modeling and analysis of the flexible robots.

Table 1: A summary of the related work and key references about the dynamic modeling and analysis of the flexible robots

\begin{tabular}{|l|l|}
\hline TOPICS & RELATED WORK AND KEY REFERENCES \\
\hline $\begin{array}{l}\text { One link flexible } \\
\text { robot }\end{array}$ & $\begin{array}{l}\text { Li et al., 1998; Endo \& Kawasaki, 2014; Feliu et al., 2014; Kalker and } \\
\text { Olsder, 1987; Wang and Wei, 1987; Wang and Vidyasagar, 1992; Kwon and } \\
\text { Book, 1994; Al-Bedoor and Khulief, 1997; Marghitu and Diaconescu, 1999; } \\
\end{array}$ \\
\hline
\end{tabular}




\begin{tabular}{|c|c|}
\hline $\begin{array}{l}\text { Two link flexible } \\
\text { robot }\end{array}$ & $\begin{array}{l}\text { Naganathan and Soni, 1987; Low and Vidyasagar, 1988; Pan et al., 1990; } \\
\text { Yuh and Young, 1991; Abe, 2009; Ata et al., 2012; Bolandi and } \\
\text { Esmaeilzadeh, 2011; Green and Sasiadek, 2004; Karagulle et al., 2015; } \\
\text { Khalil et al., 2017; Meghdari and Fahimi, 2001; Asada et al., } 1990\end{array}$ \\
\hline $\begin{array}{l}\text { Multi-link flexible } \\
\text { robot }\end{array}$ & $\begin{array}{l}\text { Book, 1984; Usoro, 1986; Jonker, 1990; Korayem et al., 2014; Augustynek } \\
\text { and Adamiec-Wójcik, 2014; De Luca and Siciliano, 1991; Amirouche and } \\
\text { Xie, 1993; Lin and Lewis, 1994; Chen, 2001; Subudhi and Morris, 2002; } \\
\text { Khadem and Pirmohammadi, 2003; Zhang, 2009; Korayem et al., 2012; } \\
\text { Raouf et al., 2017; Korayem et al., 2012; Korayem \& Shafei, 2013; } \\
\text { Korayem \& Shafei, } 2015\end{array}$ \\
\hline $\begin{array}{l}\text { Newton-Euler's } \\
\text { equations - } \\
\text { based approach }\end{array}$ & $\begin{array}{l}\text { Abbas et al., 2017; Naganathan \& Soni, 1987; Jonker, 1990; Wang \& Wei } \\
\text { 1987; Yuh \& Young, 1991; Boyer \& Coiffet, 1996; Augustynek \& Adamiec- } \\
\text { Wójcik, 2012; Briot \& Khalil, 2014; Khalil et al., } 2017\end{array}$ \\
\hline $\begin{array}{l}\text { Lagrange's } \\
\text { equations - based } \\
\text { approach }\end{array}$ & $\begin{array}{l}\text { Bricout et al., 1990; Book, 1984; Naganathan \& Soni 1987; Lin \& Lewis, } \\
\text { 1994; Usoro et al., 1986; Kalker \& Olsder, 1987; Low \& Vidyasagar, 1988; } \\
\text { Changizi \& Shabana, 1988; Lin \& Lewis, 1994; Pan et al., 1990; Asada et } \\
\text { al., 1990; De Luca \& Siciliano, 1991; Wang \& Vidyasagar, 1992; Al-Bedoor } \\
\text { \& Khulief, 1997; Tokhi et al., 2001; Chen, 2001; Subudhi, \& Morris, 2002; } \\
\text { Zhang, 2009; Abe, 2009; Ata et al., 2012; Raouf et al., 2017; Hewit, 1997; } \\
\text { Mehrez \& El-Badawy, 2010; Choi et al., 1998; Tarvirdizadeh \& Yousefi- } \\
\text { Koma, 2012; Damaren, 2000; Marghitu \& Diaconescu, 1999, and Green \& } \\
\text { Sasiadek, } 2004\end{array}$ \\
\hline $\begin{array}{l}\text { Kane's equations } \\
\text { - based approach }\end{array}$ & Amirouche \& Xie, 1993; Meghdari \& Fahimi, 2001 \\
\hline $\begin{array}{l}\text { Gibbs-Appell's } \\
\text { equations - based } \\
\text { approach }\end{array}$ & $\begin{array}{l}\text { Khadem \& Pirmohammadi, 2003; Korayem et al., 2014; Korayem et al., } \\
\text { 2012; Korayem \& Shafei, 2013; Korayem \& Shafei, } 2015\end{array}$ \\
\hline $\begin{array}{l}\text { Hamilton's } \\
\text { equations - based } \\
\text { approach }\end{array}$ & Pratiher \& Dwivedy, 2007; Ju et al., 2016 \\
\hline $\begin{array}{l}\text { Finite element } \\
\text { method - based } \\
\text { formulation }\end{array}$ & $\begin{array}{l}\text { Du et al., 1996; Płosa \& Wojciech, 2000; Jonker, 1990; Naganathan \& Soni, } \\
\text { 1987; Tokhi et al., 2001; Al-Bedoor, \& Almusallam, 2000; Mahto, 2014; } \\
\text { Karagulle et al., 2015, 2017; Al-Bedoor \& Khulief, 1997; Pan et al., 1990; } \\
\text { Wang \& Mills, 2006; Usoro, 1990; Geradin \& Cardona, 2001; Heidari et al., } \\
2013\end{array}$ \\
\hline $\begin{array}{l}\text { Assumed mode } \\
\text { method - based } \\
\text { formulation }\end{array}$ & $\begin{array}{l}\text { Hewit, 1997; Mehrez \& El-Badawy, 2010; Choi et al., 1998; Book, 1984; De } \\
\text { Luca \& Siciliano, 1991; Lin \& Lewis, 1994; Chen, 2001; Subudhi \& Morris, } \\
\text { 2002; Zhang, 2009; Raouf et al., 2017; Wang \& Vidyasagar, 1992; } \\
\text { Tarvirdizadeh \& Yousefi-Koma, 2012; Low \& Vidyasagar, 1998; Abe, } \\
\text { 2009; Ata et al., 2012; Damaren, 2000; Marghitu \& Diaconescu, 1999; } \\
\text { Green \& Sasiadek, 2004; Khadem \& Pirmohammadi, 2003; Korayem et al, } \\
\text { 2014; Ju et al., 2016; Shafei \& Shafei, 2018; Li \& Sankar, } 1992\end{array}$ \\
\hline $\begin{array}{l}\text { Implementation } \\
\text { of flexible robots } \\
\text { and experiments }\end{array}$ & $\begin{array}{l}\text { Massoud, Naraghi \& Mahzoon , 2018; Korayem et al., 2014; Luo, 1993; } \\
\text { Shafei \& Korayem, 2017; Nagaraj, Nataraju \& Ghosal, 1997; Yim, Zuang \& } \\
\text { Singh, 1993; Korayem \& Dehkordi, } 2018\end{array}$ \\
\hline
\end{tabular}

The contents and a number of the research about the one-link flexible robot dynamics are huge. The Lagrange's equations were used by Kalker \& Olsder (1987) and Li \& Sankar (1992) to derive the 
equations of motion of flexible robot and driven mechanisms with flexible links. The dynamic equations were derived by Wang and Vidyasagar (1992) for a class of multilink manipulators with the last link flexible. Kwon and Book (1994) reported a method to solve the inverse dynamic problem in time domain. Al-Bedoor and Khulief (1997) and Tokhi et al. (2001) emphasized on the finite element formulation of dynamic equations, and Ju et al. (2016) and Marghitu \& Diaconescu (1999) utilized AMM for the dynamic modelling.

There has been a lot of efforts working on the dynamic modeling and analysis of two-link flexible robots. However, most of these studies focused on the flexible robots with two revolute joints. Pan et al. (1990) investigated the modeling of the flexible robot with the fixed prismatic joints, in which the axial shortening effect of the link was taken into accounts. Yuh and Young (1991) studied a beam that has a rotational and translational motion, in which the time-varying partial differential equation and the boundary conditions were derived to describe the lateral deflection of the beam.

Recently, there has been a number of studies that focus on the dynamic modeling and analysis of the multi-link flexible robots and the related robotic mechanisms. However, most of these studies emphasised on the flexible robots with the use of the revolute joints. As discussed and mentioned in Section 1, there was a few studies (Korayem et al., 2014; Khadem \& Pirmohammadi, 2003) that proposed the methods for modeling of a multi-link flexible robot that uses the prismatic joints. However, the study done by Korayem et al. (2014) only considered the case in which each arm link of the given length rotates and reciprocates through a rigid joint. The work done by Khadem \& Pirmohammadi (2003) focused on the sliding prismatic joint; however, the elasticity effects that involve in the time-varying value of a link length, associated with the use of all types of the prismatic joint, have not been investigated. Basically, the generalized methods and techniques for the kinematic and dynamical modelling of the flexible robot that consists of the revolute, fixed prismatic and sliding prismatic joints have not been well studied.

Apart from the works investigating different architectures of flexible robot, there has also been a wide spectrum of studies focusing on various analytical approaches, incorporated with FEM or AMM, for the dynamic modelling of the multi-link flexible robot as shown in Table 1. As previously mentioned, FEM has been applied for decades to model and analyse complex structural engineering problems. Using FEM, Jonker (1990) addressed the Newton Euler dynamic model for the flexible robot using the rotary joins, with the use of separate rigid body displacements and actual deformations to describe the current state of a finite element. In FEM, the behavior of the elasticity of a flexible robot arm is observed on a rigid body motion in which the elastic deformation is then superimposed. FEM was also used for the dynamic modelling applications. Du et al., (1996) presented the general nonlinear dynamics model which was developed for three-dimensional (3D) flexible robots. Each link is discretized by the finite elements with its inertia lumped at the nodes of each element. Naganathan and Soni (1987) investigated the coupling effects of kinematics and flexibility in the flexible robots. Augustynek \& Adamiec-Wójcik (2012) studied the mechanism with the flexible beam-like links which use the revolute joints. Usoro et al. (1986) used the Lagrangian approach for the mathematical modeling of the lightweight flexible robots. Pan et al. (1990) developed a general dynamic model for design and control of the flexible robots which use the prismatic joints. Tokhi et al. (2001) presented the theoretical and experimental investigations into the dynamic modelling and characterisation of a flexible robotic system. Al-Bedoor \& Khulief (1997) formulated the dynamic model of a sliding link with a prismatic joint. Wang \& Mills (2006) presented a finite element - Lagrangian formualtion of dynamic equations for a flexible-link planar parallel platform. By using FEM and Kane's equations, Amirouche \& Xie (1993) addressed a matrix formulation of the dynamical equations for the flexible multibody systems. Heidari et al. (2013) investigated a nonlinear finite element model for the dynamic analysis of three-dimensional flexible link manipulators, in which both the geometric elastic nonlinearity and the foreshortening effects are considered. The use of FEM approach for the dynamic modelling of flexible multibody systems was discussed by Geradin \& Cardona (2001). Lochan et al. (2016) discussed advantages of FEM in dynamic modelling of a flexible robot, and it was showed that the main advantage of FEM over the other methods is that the connections in FEM are supposed to be clamped free, with at least two modes shape per link. Another advantage of FEM over analytical 
solution methods is that it is capable of handling nonlinear conditions easily. FEM can also handle irregularities in the structure and mixed boundary conditions. Meanwhile, AMM has long been applied as well for the dynamic modelling and analysis of the flexible robots, in which the link elasticity is usually modelled by a truncated finite modal series, in terms of the spatial mode eigenfunctions and time-varying mode amplitudes. In particular, Shafei \& Shafei (2018) have employed AMM approach for the dynamic modeling of the oblique impact of a multi-flexible-link robotic manipulator. It is noted that the dynamic modelling of the flexible robots also needs to be considered when working on the control law design. Matsuno et al. (1987) analysed the AM - Lagrangian dynamic model to develop the closed-loop feedback control laws for a single link flexible robot. Bolandi \& Esmaeilzadeh (2011) presented an exact tip trajectory tracking control of a flexible robot, based on the tip position and the strain gauge measurements through the nonlinear control design. Based on Hamilton's eqautions, Zhang et al. (2016) formulated the dynamic equations to control vibrations of the one-link flexible robot.

For validation of the dynamic modelling and the control design, there has been a number of researches conducting experiments on actual flexible robots. Massoud et al. (2018), Korayem et al. (2014) and Luo (1993) designed and conducted experiments for one link flexible robot. Experiments for multi-link flexible robots were implemented by Shafei \& Korayem (2017), Nagaraj et al. (1997), Yim et al. (1993), and Korayem \& Dehkordi (2018). In particular, Korayem \& Dehkordi (2018) presented experiments for a mobile manipulator, with two flexible links and revolute-prismatic joints to demonstrate the performance of a dynamic model formulated by themself.

\section{Kinematics of a planar flexible robot}

In this section, the kinematic model of a multi-link flexible robot is formulated and analysed. Specifically, let us consider the case of a planar flexible robot consisting of $n$ links and $n$ joints. It is assumed that the elastic deformation of a link is small. Each link is treated as an assemblage of a finite number of beam elements.

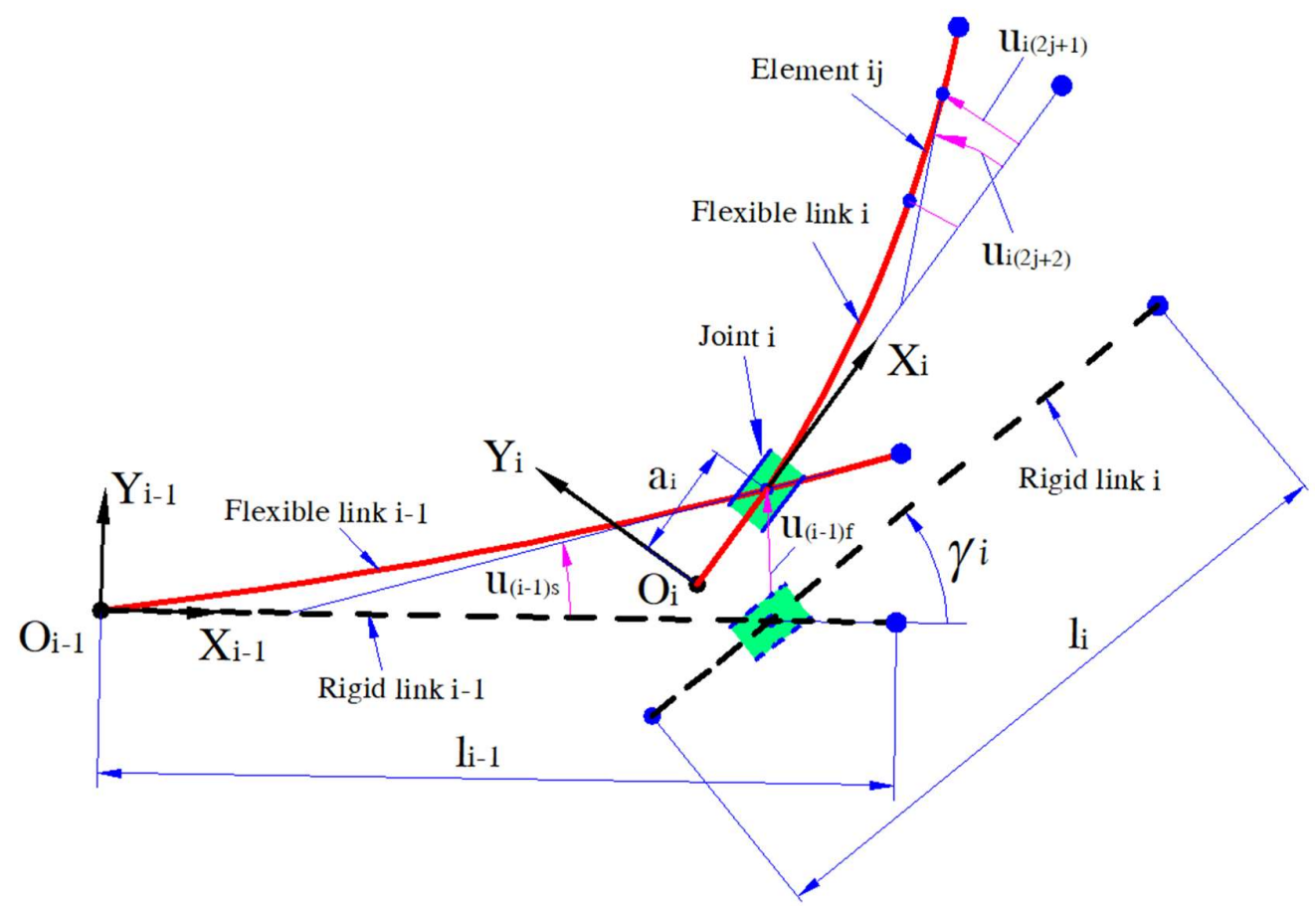

Fig. 2. A generalized schematic of an arbitrary pair of flexible links: a nominal \& actual configuration. 
Fig. 2 presents a generalized schematic of an arbitrary pair of flexible links of a planar flexible robot, with an arbitrary couple of links, namely link $i-1$ and link $i$. A link $i-1$ connects with a link $i$ by a joint $i$ which can be the following three joint types: Revolute joints $(R)$, Sliding prismatic joints $\left(P_{a}\right)$ and Fixed prismatic joints $\left(P_{b}\right)$. The link $i$ with a length $l_{i}$ is divided into $n_{i}$ elements of the equal length $l_{i e}$. Each element $j$ of the link $i$ (element $i j$ ) has two nodes. Each node has a flexural displacement and a flexural slope. For the element $i j, \mathbf{p}_{i j}=\left[\begin{array}{llll}u_{(i) 2 j-1} & u_{(i) 2 j} & u_{(i) 2 j+1} & u_{(i) 2 j+2}\end{array}\right]^{T}$ is the vector of the flexural displacement and flexural slope. In Fig.2, $u_{(i-1) f}$ and $u_{(i-1) s}$ are the flexural displacement and slope at the nominal articulation point between two links. If the joint $i$ is a revolute joint or a fixed prismatic joint, the point is at the distal end of link $i-1$, and $u_{(i-1) f}=u_{(i-1)\left(2 n_{i-1}+1\right)}$ and $u_{(i-1) s}=u_{(i-1)\left(2 n_{i-1}+2\right)}$. If the joint $i$ is a sliding prismatic joint, the point is at the distal end of the sliding element $k$ of the link $i-1$ through the joint, and $u_{(i-1) f}=u_{(i-1)(2 k+1)}$ and $u_{(i-1) s}=u_{(i-1)(2 k+2)}$.

Let us define $O_{i} \equiv\left(O_{i} X_{i} Y_{i} Z_{i}\right)$ as the local coordinate system attached to the link $i$, where the origin $O_{i}$ is fixed to the proximal end of the link $i$ and the axis $X_{i}$ points in the direction of the link $i$. Similarly, $O_{i-1} \equiv\left(O_{i-1} X_{i-1} Y_{i-1} Z_{i-1}\right)$ is defined for the link $i-1 . O_{0} \equiv\left(O_{0} X_{0} Y_{0} Z_{0}\right)$ is the reference coordinate system fixed to the base.

Lemma: The general homogeneous transformation matrix $\boldsymbol{\Theta}_{(i-1) i}$ describing the kinematics of both the motion of a joint $i$ and the deformation of a link $i-1$ can be written in the following form:

$$
\boldsymbol{\Theta}_{(i-1) i}=\left[\begin{array}{cccc}
\cos \left(\vartheta_{i}+u_{(i-1) s}\right) & -\sin \left(\vartheta_{i}+u_{(i-1) s}\right) & 0 & \chi_{i}+a_{i} \cos \left(\vartheta_{i}+u_{(i-1) s}\right) \\
\sin \left(\vartheta_{i}+u_{(i-1) s}\right) & \cos \left(\vartheta_{i}+u_{(i-1) s}\right) & 0 & u_{(i-1) f}+a_{i} \sin \left(\vartheta_{i}+u_{(i-1) s}\right) \\
0 & 0 & 1 & 0 \\
0 & 0 & 0 & 1
\end{array}\right]
$$

where $\chi_{i}, \vartheta_{i}, u_{(i-1) s}, u_{(i-1) f}$, and $a_{i}$ are symbolic notations representing the joint variables and parameters according to the joint types as shown in Table 2.

Table 2: Joint variables and parameters of a link

\begin{tabular}{lcccccc}
\hline Joint Type & $\chi_{i}$ & $\vartheta_{i}$ & $u_{(i-1) s}$ & $u_{(i-1) f}$ & $a_{i}$ & Note \\
\hline$R$ & $l_{i-1}$ & $\theta_{i}^{*}$ & $u_{(i-1)\left(2 n_{i-1}+2\right)}$ & $u_{(i-1)\left(2 n_{i-1}+1\right)}$ & 0 & $\begin{array}{l}(*): \text { joint } \\
\text { variable }\end{array}$ \\
$P_{a}$ & $d_{i}^{*}$ & $\gamma_{i}$ & $u_{(i-1)(2 k+2)}$ & $u_{(i-1)(2 k+1)}$ & 0 & $k \in\left\{1,2,3, \ldots, n_{i-1}\right\}$ \\
$P_{b}$ & $l_{i-1}$ & $\gamma_{i}$ & $u_{(i-1)\left(2 n_{i-1}+2\right)}$ & $u_{(i-1)\left(2 n_{i-1}+1\right)}$ & $d_{i}^{*}-l_{i}$ & \\
\hline
\end{tabular}

Proof: In order to obtain the coordinate system $O_{i} X_{i} Y_{i} Z_{i}$, from $O_{i-1} X_{i-1} Y_{i-1} Z_{i-1}$, the following translations and rotations are performed. 
(i) A pure translation of the coordinate system $O_{i-1}$ along $\chi_{i}$ in the direction $X_{i-1}$. The translating distance $\chi_{i}$ will be the joint variable $d_{i}$, if the joint $i$ is a sliding prismatic joint. Otherwise, $\chi_{i}$ is the length $l_{i-1}$ of the link $i-1$. The homogeneous transformation matrix characterizing this transformation is denoted as $\mathbf{T}\left(\chi_{i}\right)$.

(ii) A translation of $O_{i-1}$, at the previous location, a long $u_{(i-1) f}$ in the direction $Y_{i-1}$. If the joint $i$ is a sliding prismatic joint, the translating distance $u_{(i-1) f}$ will be the flexural displacement $u_{(i-1)(2 k+1)}$ calculated at the distal node of an element $k$ of the link $i-1$. At a time instance, this element currently slides inside the joint $i$. Otherwise, it is equal to the flexural displacement at the tip of the link $i-1, u_{(i-1)\left(2 n_{i-1}+1\right)}$. The homogeneous transformation matrix describing this translation is denoted as $\mathbf{T}\left(u_{(i-1) f}\right)$.

It is noted that, since the small elastic deformations are assumed, the translation (ii) is also assumed to be carried out before the next rotation (iii).

(iii) A rotation of $O_{i-1}$, at the previous location, around $Z_{i-1}$. If the joint $i$ is a sliding prismatic joint, the rotation angle $u_{(i-1) s}$ will be the flexural slope $u_{(i-1)(2 k+2)}$ calculated at the distal node of the element $k$ of the link $i-1$. At a time instance, this element currently slides inside the joint $i$. Otherwise, it is equal to the flexural slope at the tip of the link $i-1, u_{(i-1)\left(2 n_{i-1}+2\right)}$ The homogeneous transformation matrix representing this rotation is $\mathbf{R}\left(u_{(i-1) s}\right)$.

(iv) A rotation of $O_{i-1}$, at the previous location, around $Z_{i-1}$. If the joint $i$ is a revolute joint, the rotation angle $\vartheta_{i}$ will be the joint variable $\theta_{i}$. Otherwise, it is the geometric angle $\gamma_{i}$ between the link $i-1$ and the link $i$. The homogeneous transformation matrix is $\mathbf{R}\left(\vartheta_{i}\right)$.

(v) A translation of $O_{i-1}$, at the previous location, back to the proximal end of the link $i$. If the joint $i$ is a fixed prismatic joint, $a_{i}=d_{i}-l_{i}$, where $d_{i}$ is the joint variable. Otherwise, it is assumed that $a_{i}=0$. The homogeneous transformation matrix is $\mathbf{T}\left(a_{i}\right)$.

Consequently, the cumulative transformation matrix is calculated as follows:

$$
\begin{gathered}
\boldsymbol{\Theta}_{(i-1) i}=\mathbf{T}\left(\chi_{i}\right) \mathbf{T}\left(u_{(i-1) f}\right) \mathbf{R}\left(u_{(i-1) s}\right) \mathbf{R}\left(\vartheta_{i}\right) \mathbf{T}\left(a_{i}\right) . \\
\boldsymbol{\Theta}_{(i-1) i}=\left[\begin{array}{cccc}
\cos \left(\vartheta_{i}+u_{(i-1) s}\right) & -\sin \left(\vartheta_{i}+u_{(i-1) s}\right) & 0 & \chi_{i}+a_{i} \cos \left(\vartheta_{i}+u_{(i-1) s}\right) \\
\sin \left(\vartheta_{i}+u_{(i-1) s}\right) & \cos \left(\vartheta_{i}+u_{(i-1) s}\right) & 0 & u_{(i-1) f}+a_{i} \sin \left(\vartheta_{i}+u_{(i-1) s}\right) \\
0 & 0 & 1 & 0 \\
0 & 0 & 0 & 1
\end{array}\right] \mathbf{~}
\end{gathered}
$$


By using the matrices $\boldsymbol{\Theta}_{(i-1) i}$, the kinematics of the flexible arm can be systematically determined. Given a value of $x, x \in\left[0, l_{i e}\right]$, on the element $j$ of the link $i$, in the local frame $O_{i}$, the point $\mathbf{r}_{i j}(x)$ can be determined as follows:

$$
\mathbf{r}_{i j}(x)=\left[\begin{array}{c}
(j-1) l_{i e}+x \\
w_{i j}(x) \\
0 \\
1
\end{array}\right]
$$

where the flexural displacement at the point $x$ is determined as follows:

$$
w_{i j}(x)=\sum_{m=1}^{4} \phi_{m}(x) u_{(i)(2 j-2+m)} \text {. }
$$

By using the transformation matrices $\boldsymbol{\Theta}_{(i-1) i}(i=1 \div n)$, in the reference frame $O_{0}$, the vector $\mathbf{r}_{0 i j}(x)$ can be calculated as follows:

$$
\mathbf{r}_{0 i j}(x)=\boldsymbol{\Theta}_{0 i} \mathbf{r}_{i j}(x)
$$

where

$$
\boldsymbol{\Theta}_{0 i}=\boldsymbol{\Theta}_{01} \boldsymbol{\Theta}_{12} \ldots \boldsymbol{\Theta}_{(i-1) i}
$$

To demonstrate the kinematic modelling as proposed, all nine $\left(3^{2}\right)$ feasible configurations of a generalized two-link flexible robot that consists of all three joint types are presented in details in Appendix. For all configurations, the proposed transformation matrix is applied to describe the kinematics of the robots. The position of the distal end of the first link and the arm tip are calculated for both the flexible robot and the rigid robot of the same geometric parameters. The following are the main summaries from the above calculations and analyses: (a) if the elastic deformation of the links is ignored, the kinematic behavior of a flexible robot and the corresponding rigid robot will be the same; (b) applying the proposed matrix, a kinematic modelling process for a flexible robot is quite familiar and common in the field of robotics; and (c) the proposed matrix is applicable to any planar flexible robots.

\section{Dynamics of a planar flexible robot}

In this section, the dynamic modelling of the general planar flexible robot is presented. The equations of motion are written by applying the Lagrangian formulation. Particularly, two new recursive formulations are introduced to formulate the global mass and stiffness matrices respectively.

In the formulation of the global mass matrix, the velocity of a mass point on an arbitrary element of a link $i$ is calculated via Jacobian matrix and the generalized velocities. The elemental kinetic energy is thus calculated and expressed in terms of the entire generalized velocities. Hence, the number of rows and the number of columns of every elemental mass matrix equals to the number of the generalized coordinates.

In the formulation of the global stiffness matrix, a vector of extended shape functions is defined. The size of this vector equals to the size of the vector of generalized coordinates. The flexural 
displacement of a point on an arbitrary element of a link $i$ can be calculated via the defined vector. Thus, the elemental potential energy is expressed in terms of the entire generalized coordinates, and the number of rows and the number of columns of every elemental stiffness matrix equals to the number of the generalized coordinates.

Consequently, the global mass and stiffness matrices can be formulated by summing over all the elemental mass and stiffness matrices of the same size, respectively.

Based on the Finite Elements - Lagrangian approach, the equation of motions for the flexible robot can be expressed as follows:

$$
\mathbf{M}(\mathbf{q}) \ddot{\mathbf{q}}+\mathbf{C}(\mathbf{q}, \dot{\mathbf{q}}) \dot{\mathbf{q}}+\mathbf{K q}+\mathbf{G}(\mathbf{q})=\mathbf{F}
$$

The equation is written with respect to the global vector of the generalized coordinates, $\mathbf{q}=\left[\begin{array}{lllllll}p_{1} & \boldsymbol{\kappa}_{1}^{T} & p_{2} & \boldsymbol{\kappa}_{2}^{T} & \ldots & p_{n} & \boldsymbol{\kappa}_{n}^{T}\end{array}\right]^{T}$, where $p_{i}$ is variable of a joint $i$, and $\boldsymbol{\kappa}_{i}=\left[\begin{array}{lllll}u_{(i) 1} & u_{(i) 2} & \ldots & u_{(i)\left(2 n_{i}+1\right)} & u_{(i)\left(2 n_{i}+2\right)}\end{array}\right]^{T}$ is the vector of the elastic displacements of all elements on a link $i$. The vector $\mathbf{q}$ includes $n$ joint variables $p_{1}, p_{2}, \ldots, p_{n}$ and $\sum_{i=1}^{n}\left(2 n_{i}+2\right)$ are the total nodal displacements and slopes of all elements on all links. Thus, the size of $\mathbf{q}$ is $\left(n+\sum_{i=1}^{n}\left(2 n_{i}+2\right)\right) \times 1$.

$$
\mathbf{F}=\left[\begin{array}{lllllll}
\tau_{1} & \underbrace{\begin{array}{lllll}
0 & \ldots & 0
\end{array}}_{2 n_{1}+2} & \ldots & \tau_{n} & \underbrace{0}_{2 n_{n}+2} \quad \ldots & 0
\end{array}\right]^{T} \text { is the vector of the applied forces/torques imposing }
$$

on $n$ joints.

The size of the global matrices $\mathbf{M}, \mathbf{C}$ and $\mathbf{K}$ is $\left(n+\sum_{i=1}^{n}\left(2 n_{i}+2\right)\right) \times\left(n+\sum_{i=1}^{n}\left(2 n_{i}+2\right)\right)$.

\subsection{Recursive formulation of the global mass matrix}

Consider an element $j$ of a link $i$, the kinetic energy of the element is computed as follows:

$$
T_{i j}=\frac{1}{2} \int_{0}^{l_{i e}} m_{i} \dot{\mathbf{r}}_{0 i j}^{T} \dot{\mathbf{r}}_{0 i j} d x
$$

In the reference frame, the velocity of the mass point located at $\mathbf{r}_{0 i j}(x)$ can be computed as follows:

$$
\dot{\mathbf{r}}_{0 i j}=\frac{\partial \mathbf{r}_{0 i j}}{\partial \mathbf{q}} \dot{\mathbf{q}}
$$

Thus, 


$$
T_{i j}=\frac{1}{2} \dot{\mathbf{q}}^{T}\left(\int_{0}^{l_{i e}} m_{i} \mathbf{J}_{i j}^{T} \mathbf{J}_{i j} d x\right) \dot{\mathbf{q}}=\frac{1}{2} \dot{\mathbf{q}}^{T} \mathbf{M}_{i j} \dot{\mathbf{q}}
$$

where Jacobian matrix is presented as follows:

$$
\mathbf{J}_{i j}=\frac{\partial \mathbf{r}_{0 i j}}{\partial \mathbf{q}} .
$$

Consequently, the elemental mass matrix for an element $j$ of a link $i$ can be written as follows:

$$
\mathbf{M}_{i j}=\left[m_{s p}\right]_{i j}=\int_{0}^{l_{i e}} m_{i} \mathbf{J}_{i j}^{T} \mathbf{J}_{i j} d x
$$

The size of matrix $\mathbf{M}_{i j}$ is $\left(n+\sum_{i=1}^{n}\left(2 n_{i}+2\right)\right) \times\left(n+\sum_{i=1}^{n}\left(2 n_{i}+2\right)\right)$.

The indexes are presented as follows: $s, p=1 \div\left(n+\sum_{i=1}^{n}\left(2 n_{i}+2\right)\right)$.

It is noted that for all $s$ and $p$ from $\left(i+2 j-1+\sum_{u=1}^{i-1}\left(2 n_{u}+2\right)\right)$ to $\left(i+2 j+1+\sum_{u=1}^{i-1}\left(2 n_{u}+2\right)\right)$,

$$
m_{s p}=\frac{m_{i} l_{i e}}{420}\left[\begin{array}{cccc}
156 & 22 l_{i e} & 54 & -13 l_{i e} \\
22 l_{i e} & 4 l_{i e}^{2} & 13 l_{i e} & -3 l_{i e}^{2} \\
54 & 13 l_{i e} & 156 & -22 l_{i e} \\
-13 l_{i e} & -3 l_{i e}^{2} & -22 l_{i e} & 4 l_{i e}^{2}
\end{array}\right]
$$

The total kinetic energy of the robot system is presented as follows:

$$
T=\sum_{i=1}^{n} \sum_{j=1}^{n_{i}} T_{i j}=\frac{1}{2} \dot{\mathbf{q}}^{T} \mathbf{M} \dot{\mathbf{q}}^{T}
$$

Finally, the global mass matrix is obtained as follows:

$$
\mathbf{M}=\sum_{i=1}^{n} \sum_{j=1}^{n_{i}} \mathbf{M}_{i j}
$$

The recursive algorithm for the symbolic formulation of the global mass matrix $\mathbf{M}$ is shown in Fig. 3. The inputs of the formulation are the number of links $n$, the number of finite elements on the links $n_{1}, n_{2}, \ldots n_{n}$, and the other parameters of the robot system. The global matrices are initiated as zero matrices. For every element $j$ of a link $i$, the position vector $\mathbf{r}_{0 i j}(x)$ is determined with Eq (5), the Jacobian $\mathbf{J}_{i j}$ is implemented with Eq (11), and the elemental mass matrix $\mathbf{M}_{i j}$ is calculated with Eq (12). For all elements on all links, the above steps are recursively looped to archive all $\mathbf{M}_{i j}$. Finally, the global matrix $\mathbf{M}$ is obtained with Eq (15). 


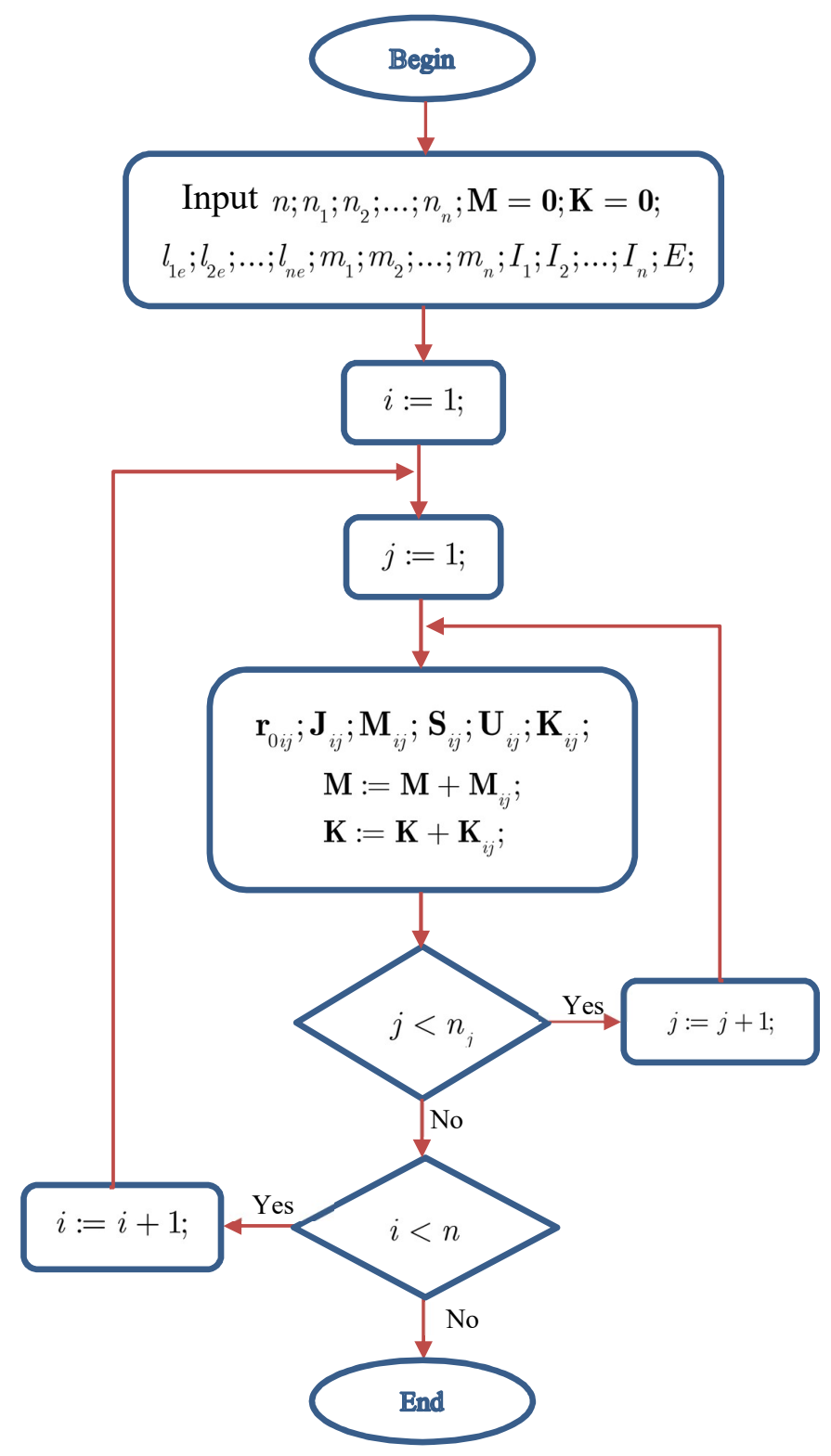

Fig. 3. A flowchart of the algorithm for formulation of the global mass and stiffness matrices

Notice that the symbolic formulation of $\mathbf{M}_{i j}$ and $\mathbf{M}$ can be effectively implemented by using a few syntaxes in the symbolic computing environments. For example, in Maple, a software developed by Waterloo Maple, two syntaxes Jacobian and int are needed for the Jacobian matrix formulation, $\mathbf{J}_{i j}=\frac{\partial \mathbf{r}_{0 i j}}{\partial \mathbf{q}}$, and the integration $\mathbf{M}_{i j}=\int_{0}^{l_{i e}} m_{i} \mathbf{J}_{i j}^{T} \mathbf{J}_{i j} d x$, respectively. .

\subsection{Recursive formulation of the global stiffness matrix}

The elastic potential energy of element $j$ of link $i$ is calculated as:

$$
P_{i j}=\frac{1}{2} \int_{0}^{l_{i e}} E I_{i}\left(\frac{\partial^{2} w_{i j}(x)}{\partial x^{2}}\right)^{2} d x
$$


Based on the vector of shape functions $\mathbf{S}=\left[\begin{array}{llll}\phi_{1}(x) & \phi_{2}(x) & \phi_{3}(x) & \phi_{4}(x)\end{array}\right]^{T}$, the following notation $\mathbf{S}_{i j}$ (the vector of extended shape functions) is defined as an elemental vector of shape functions, which corresponds to an element $j$ on a link $i$. The size of $\mathbf{S}_{i j}$ equals to the size of $\mathbf{q}$ :

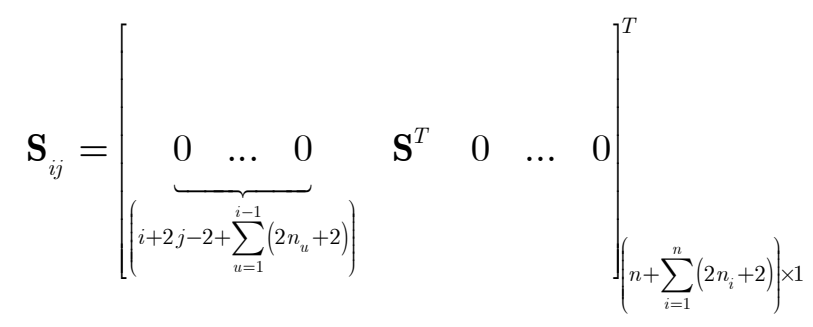

The flexural displacement $w_{i j}(x)$ expressed in Eq (4) is now recalculated with the following equation:

$$
w_{i j}(x)=\mathbf{S}_{i j}^{T} \mathbf{q}
$$

Substituting Eq (18) into Eq (16) yields the following result:

$$
\begin{gathered}
P_{i j}=\frac{1}{2} \int_{0}^{l_{i e}} E I_{i}\left(\frac{\partial^{2} \mathbf{S}_{i j}^{T}}{\partial x^{2}} \mathbf{q}\right)^{2} d x, \\
\mathbf{U}_{i j}=\left(\frac{\partial^{2} \mathbf{S}_{i j}^{T}}{\partial x^{2}}\right)
\end{gathered}
$$

Thus,

$$
P_{i j}=\frac{1}{2} \mathbf{q}^{T}\left(E I_{i} \int_{0}^{l_{i e}} \mathbf{U}_{i j}^{T} \mathbf{U}_{i j} d x\right) \mathbf{q}=\frac{1}{2} \mathbf{q}^{T} \mathbf{K}_{i j} \mathbf{q}
$$

Consequently, the elemental stiffness matrix for element $j$ of link $i$ can be written as:

$$
\mathbf{K}_{i j}=\left[k_{s p}\right]_{i j}=E I_{i} \int_{0}^{l_{i e}} \mathbf{U}_{i j}^{T} \mathbf{U}_{i j} d x
$$

The size of matrix $\mathbf{K}_{i j}$ is $\left(n+\sum_{i=1}^{n}\left(2 n_{i}+2\right)\right) \times\left(n+\sum_{i=1}^{n}\left(2 n_{i}+2\right)\right)$, and the indexes $s, p=1 \div\left(n+\sum_{i=1}^{n}\left(2 n_{i}+2\right)\right)$.

The total elastic potential energy of the robot is calculated as follows:

$$
P=\sum_{i=1}^{n} \sum_{j=1}^{n_{i}} P_{i j}=\frac{1}{2} \mathbf{q}^{T} \mathbf{K} \mathbf{q}
$$


Finally, the global stiffness matrix can be calculated as follows:

$$
\mathbf{K}=\sum_{i=1}^{n} \sum_{j=1}^{n_{i}} \mathbf{K}_{i j}
$$

The matrices $\mathbf{K}$ and $\mathbf{K}_{i j}$ have the same size of $\left(n+\sum_{i=1}^{n}\left(2 n_{i}+2\right)\right) \times\left(n+\sum_{i=1}^{n}\left(2 n_{i}+2\right)\right)$.

It is noted that, for all

$$
\text { s or } p \notin\left[\left(i+2 j-1+\sum_{u=1}^{i-1}\left(2 n_{u}+2\right)\right),\left(i+2 j+1+\sum_{u=1}^{i-1}\left(2 n_{u}+2\right)\right)\right] \text {, }
$$

the corresponding entries in matrix $\mathbf{K}_{i j}$ are equal to zero, $k_{s p}=0$. This is because $w_{i j}(x)$ calculated via $\mathrm{Eq}(18)$ depends only on four nodal elastic displacements at the two nodes of an element $j$ on a link $i$.

$$
\text { For all } s \text { and } p \in\left[\left(i+2 j-1+\sum_{u=1}^{i-1}\left(2 n_{u}+2\right)\right),\left(i+2 j+1+\sum_{u=1}^{i-1}\left(2 n_{u}+2\right)\right)\right] \text {, }
$$

the corresponding entries $k_{s p}$ are the following constants:

$$
k_{s p}=\frac{E I_{i}}{l_{i e}^{3}}\left[\begin{array}{cccc}
12 & 6 l_{i e} & -12 & 6 l_{i e} \\
6 l_{i e} & 4 l_{i e}^{2} & -6 l_{i e} & 2 l_{i e}^{2} \\
-12 & -6 l_{i e} & 12 & -6 l_{i e} \\
6 l_{i e} & 2 l_{i e}^{2} & -6 l_{i e} & 4 l_{i e}^{2}
\end{array}\right]
$$

Similar to the symbolic formulation of the global mass matrix, the recursive algorithm for the formulation of $\mathbf{K}_{i j}$ and $\mathbf{K}$ is shown in Fig. 3, and it can be implemented effectively in the symbolic computing environments.

\subsection{Boundary conditions}

Since a link $i$ is connected with the previous one via a revolute joint or a sliding prismatic joint, it is assumed that the elastic displacements at the first node of the first element of the link $i$ vanishes ( $\left.u_{(i) 1}=u_{(i) 2}=0\right)$. Therefore, such the entries $u_{(i) 1}$ and $u_{(i) 2}$ in a vector $\mathbf{q}$ are eliminated. The corresponding rows and columns in $\mathbf{M}, \mathbf{C}, \mathbf{K}$ are removed. This is a fixed boundary condition.

In the case that a link $i$ is connected with the previous one via a fixed prismatic joint, it is assumed that the elastic displacements of the element $k$ that currently slides inside the joint vanishes. The value of $k$ changes with respect to time. This is a time dependent boundary condition which is implemented in the dynamic analysis process.

In the case that a link $i$ is connected with the previous one via a sliding prismatic joint, the elastic displacements $u_{(i-1)(2 k+1)}$ and $u_{(i-1)(2 k+2)}$ of the element $k$ on link $i-1$ that currently slides inside the joint is considered as the elastic displacements of the nominal articulation point between a link $i-1$ and a link $i$. This is also a time dependent boundary condition. 


\subsection{Numerical examples of the dynamic analysis}

In this study, two robot configurations $C_{P_{a} R}$ and $C_{R P_{b}}$ with the last link flexible are modelled and simulated. The first robot $C_{P_{a} R}$ is a two-joints arm in which the first joint is a sliding prismatic joint $P_{a}$, and the second one is a revolute joint $R$. The second robot $C_{R P_{b}}$ is a two-joints arm that consists of a revolute joint $R$ and a fixed prismatic joint $P_{b}$. Simulation of both the robots of different prismatic joint sequence is to demonstrate more clearly the flexibility effect of the last link. In the first robot $C_{P_{a} R}$ , the last link is connected with the previous one via a revolute joint, meanwhile in the second robot $C_{R P_{b}}$, the last link is connected with the previous link via a prismatic joint. Structural parameters of the two robots and the inputs of applied forces/torques are shown in Table 3.

To demonstrate the flexibility effect, the simulation results of the flexible robots are compared to that of the rigid robot of the same parameters.

Table 3: Structural parameters and the inputs of applied forces/torques for simulation and analysis

\begin{tabular}{lcc}
\hline Parameters of models & $C_{P_{a} R}$ & $C_{R P_{b}}$ \\
\hline Length of Link 1 (m) & $l_{1}=0.2$ & $l_{1}=0.2$ \\
Mass of Link 1 (kg) & $m_{1}=1.4$ & $m_{1}=3.9$ \\
Angle between links (rad) & $\gamma_{1}=\pi / 2$ & $\gamma_{2}=0$ \\
Mass of payload (g) & $m_{t}=100$ & $m_{t}=100$ \\
Length of Link 2 (m) & $l_{2}=0.8$ & $l_{2}=0.8$ \\
Number of elements of Link 2 & $n=3$ & $n=5$ \\
Cross section area of Link 2 (m $\left.{ }^{2}\right)$ & $A=1.2 \times 10^{-4}$ & $A=4.5 \times 10^{-5}$ \\
Mass density of Link 2 $\left(\mathrm{kg} / \mathrm{m}^{3}\right)$ & $\rho=7850$ & $\rho=7850$ \\
Mass per meter of Link 2 $\left(\mathrm{kg} / \mathrm{m}^{3}\right)$ & $m_{2}=\rho . A=0.942$ & $m_{2}=\rho . A=0.35$ \\
Young's modulus (N/m $\left.{ }^{2}\right)$ & $E=2 \times 10^{10}$ & $E=2 \times 10^{10}$ \\
Inertial moment of a cross section (m $\left.{ }^{4}\right)$ & $I=9 \times 10^{-11}$ & $I=8.44 \times 10^{-12}$ \\
Applied forces/torques & Bang-Bang rule & Bang-Bang rule \\
Simulation time (sec) & 10 & 20 \\
\hline
\end{tabular}

The simulation results are shown in Figs. 5 to 9. With the input of Bang-bang applied torque/force presented in Fig. 4, the time evolution of the joint displacements for both the flexible robots and rigid robots are shown in Fig. 5 . After $0.5 \mathrm{sec}$, the dynamic behavior of a system mainly depends on their inertia. The flexural displacements and slopes at the end-effectors are illustrated in Fig. 6. Figs 7, 8 and 9 present the trajectories of the endpoint of the robotic arm. The calculation results show the deviation between the trajectories of the flexible and rigid robots. 


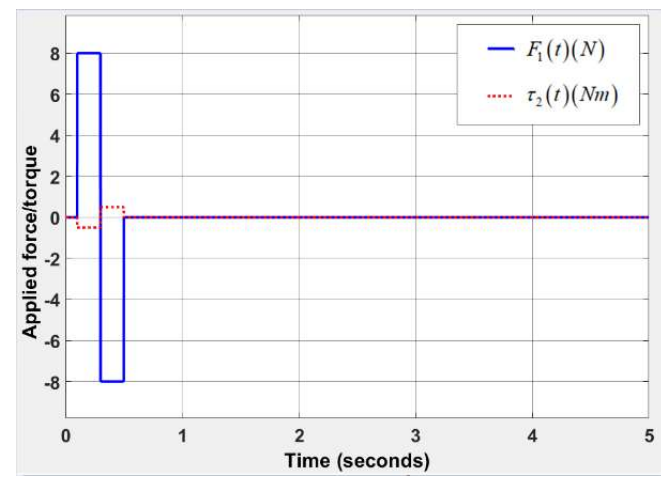

a) Applied force/torque for $C_{P_{a} R}$

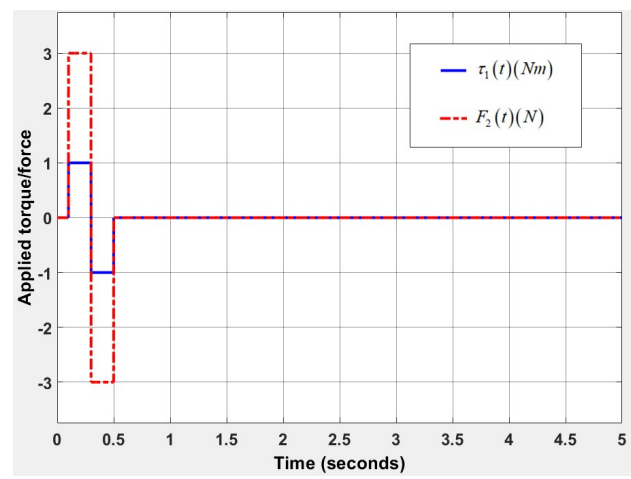

b) Applied torque/force for $C_{R P_{b}}$

Fig. 4. The Bang-bang applied force/torque

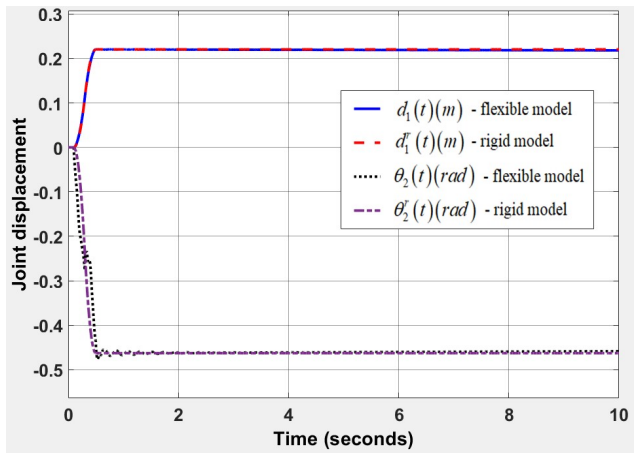

a) $C_{P_{a} R}$

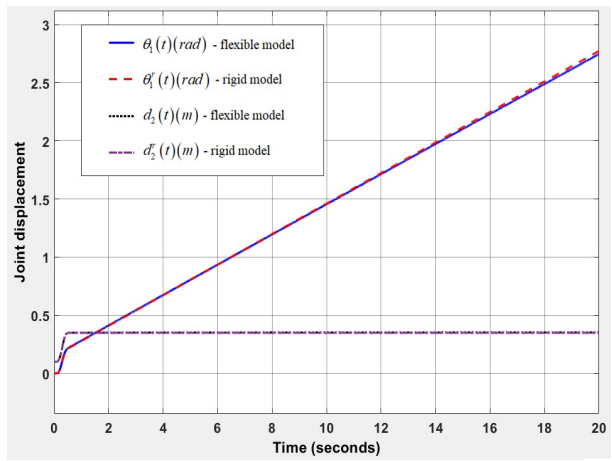

b) $C_{R P_{b}}$

Fig. 5. The joint displacements of the flexible robots and the rigid robots

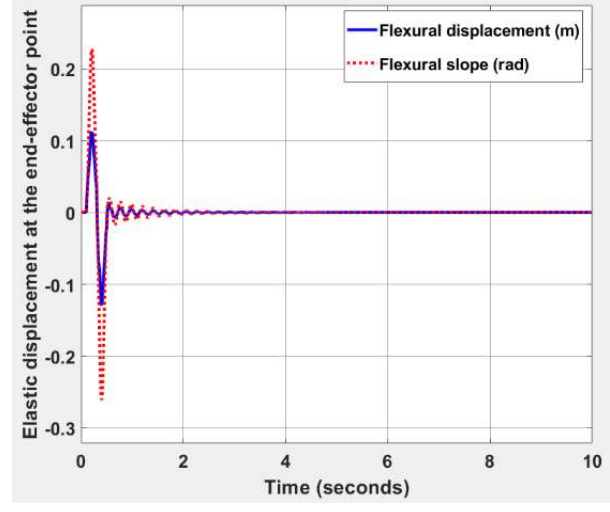

a) $C_{P_{a} R}$

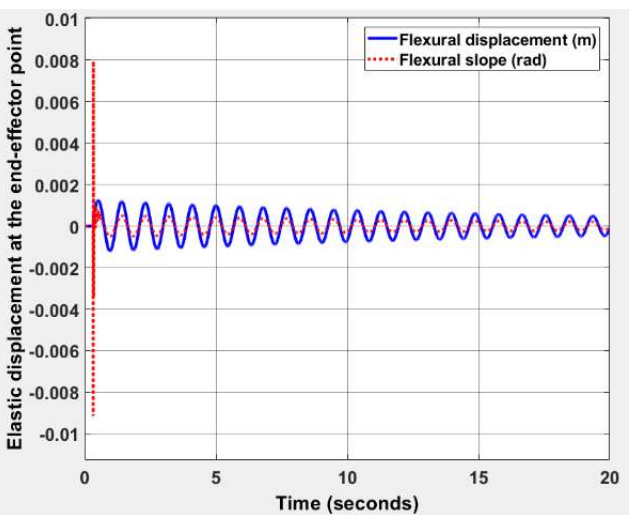

b) $C_{R P_{b}}$

Fig. 6. The flexural displacement and slope at the end-effector 


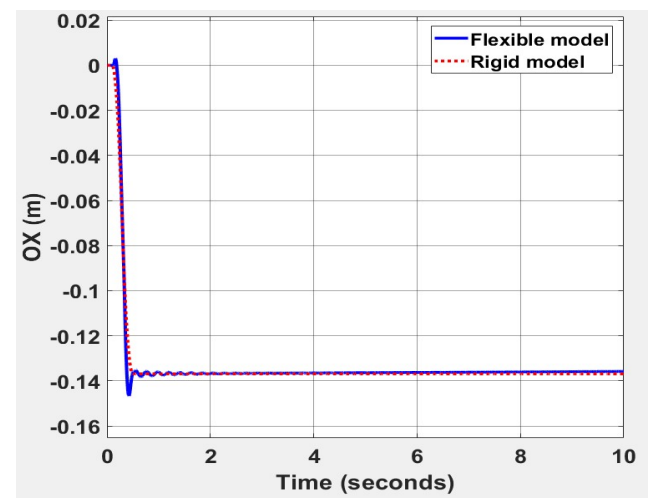

a) $C_{P_{a} R}$

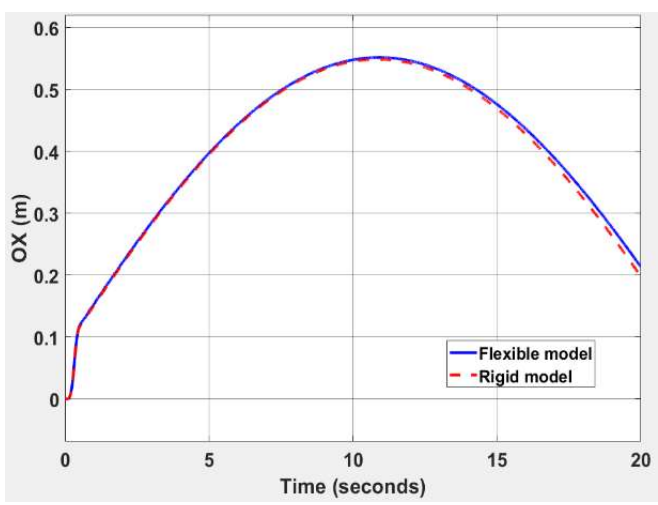

b) $C_{R P_{b}}$

Fig. 7. A trajectory of the end-effector in the OX axis

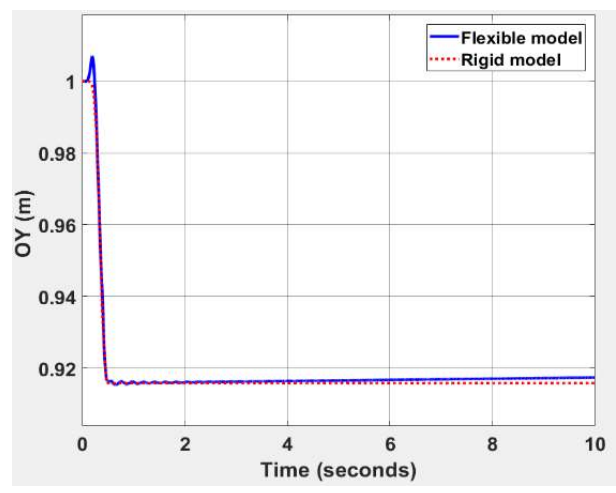

a) $C_{P_{a} R}$

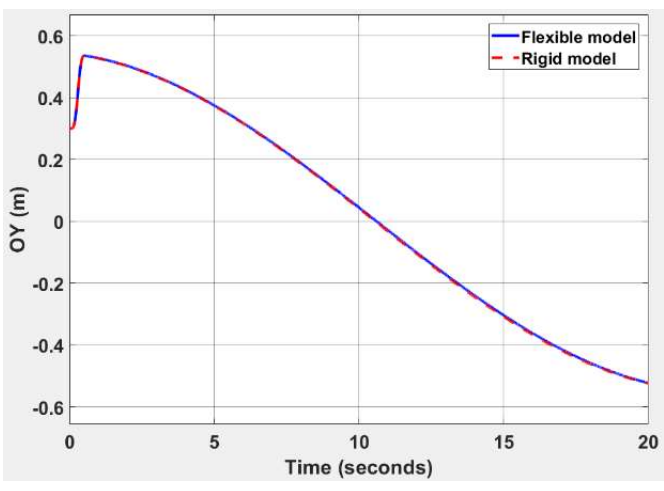

b) $C_{R P_{b}}$

Fig. 8. A trajectory of the end-effector in the OY axis

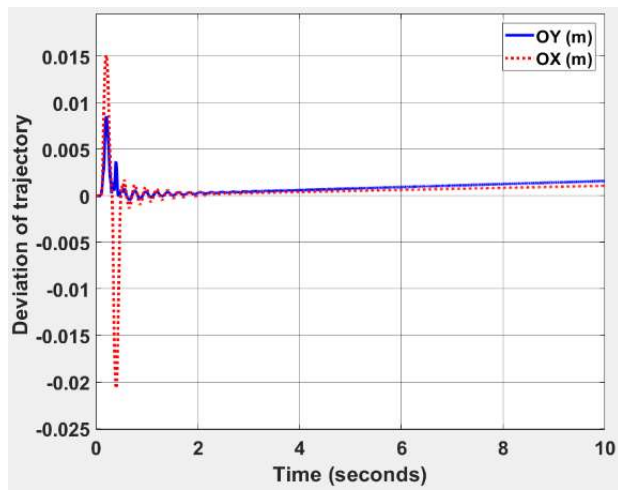

a) $C_{P_{a} R}$

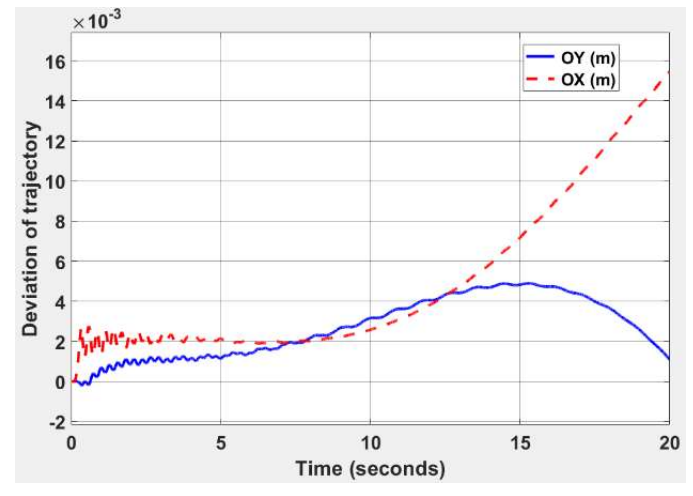

b) $C_{R P_{b}}$

Fig. 9. The deviation between the trajectories of the flexible robot and the rigid one

\section{Experiments for validation of the forward dynamic analysis results}

The experiments were designed and conducted for validation of the forward dynamic analysis results based on the proposed dynamic modelling method. 

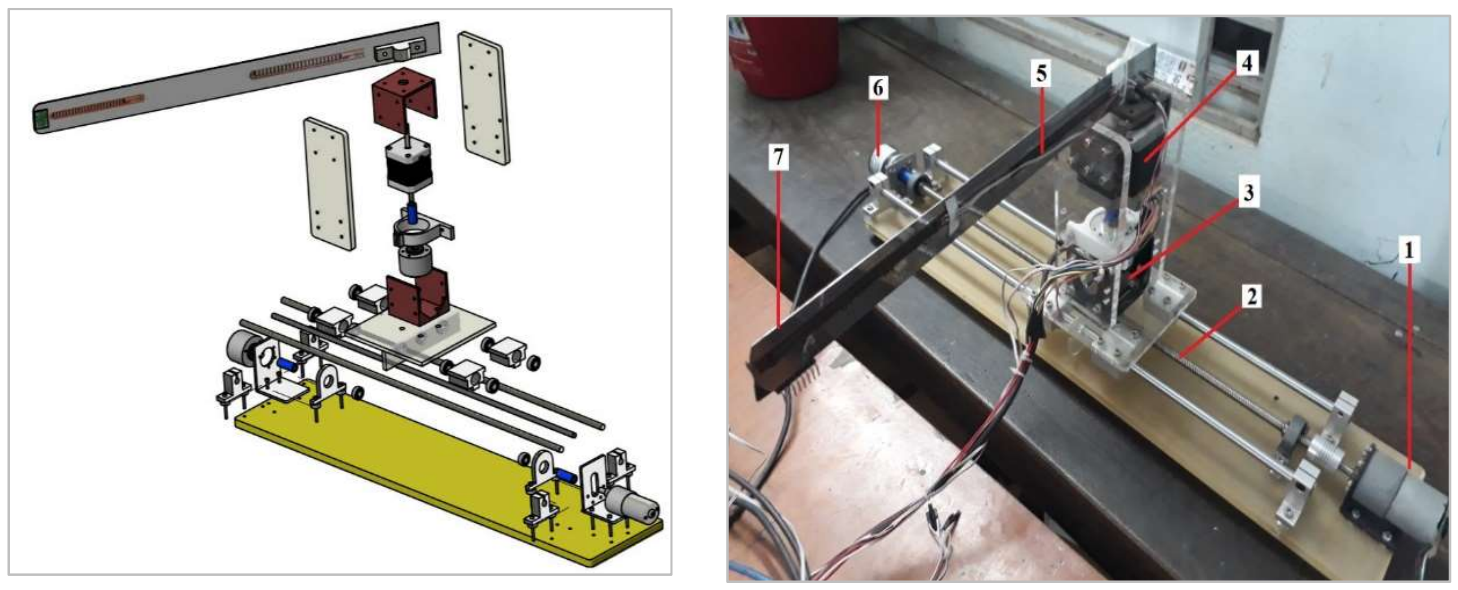

Fig. 10. A prototype of a planar flexible robot for the experimental work: (1) DC motor, (2) Lead screw, (3) Rotary encoder 1, (4) Step motor, (5) Flex sensor, (6) Rotary encoder 2, (7) Flexible link

A prototype of a robot and experimental set-ups are shown in Figs. 10 and 11. The system includes a robot, a payload, two flex sensors of 4.5-inch FSL0095-103-ST, one motor GB 37-3530 DC $12 \mathrm{~V}$, one NEMA 17 stepper motor 200 steps/rev 12V and two rotary encoders LPD3806-600BM-G5$24 \mathrm{~V}-2 \mathrm{P}-\mathrm{AB}$. A Terminal Board STM32 F407/417 is used as the main board of the control system of the robot. The STM32F407/417 offers the performance of the Cortex ${ }^{\mathrm{TM}}-\mathrm{M} 4$ core (with floating point unit) running at $168 \mathrm{MHz}$. The control program is written in KeilC programming language. The readings of the experiments are displayed in Labview software. The robot is constructed with two joints. The first joint is a prismatic joint that is actuated by the motor (1) through the lead screw (2). The second joint is a revolute joint driven by the motor (4). The robot is supposed to operates in the horizontal plane. The two flex sensors FSL0095-103-ST $112.24 \mathrm{~mm}$ in length are adhered to the surface and towards both ends of the flexible link. The input voltage of the sensors is $4.98 \mathrm{~V}$, the maximum resistance of the sensors is $110 k \Omega$. Notice that there have been some types of sensors such as the accelerometer (Khorrami et al., 1992; Martins et al., 2003) and the strain gauge (Luo, 1993) that could be used as alternatives to the flex sensor for measuring the elastic deflection of the link. However, the bend sensors have been widely used in various applications, since it is a low cost, and it is easy to use. It does not need complicated signal processing because the bend angle of the sensor is proportional to the output signal of the displacement sensing module.

Structural parameters of the robot are given in Table 2, except some parameters were changed as $L_{1}=0.005(m) ; L_{2}=0.3(m) ; m_{t}=20(g) ; A=2 \times 10^{-5}\left(m^{2}\right)$.

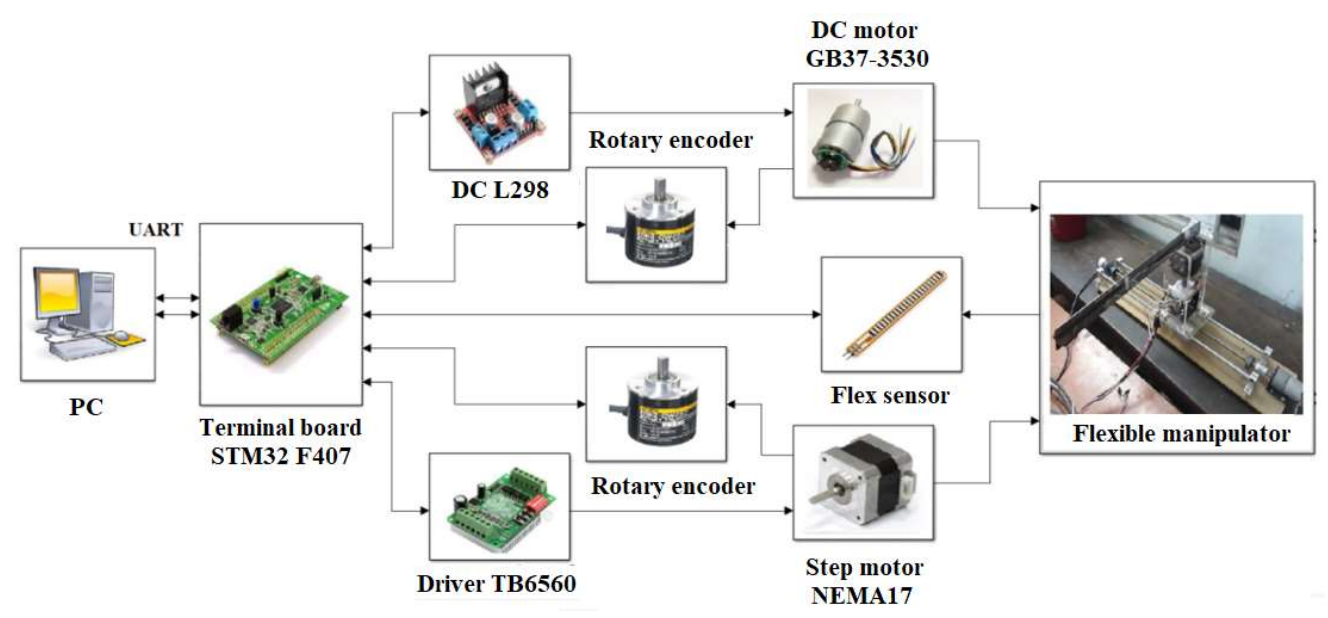

Fig. 11. A block diagram of the experimental setup for validation of the forward dynamic analysis results 
The Bang-bang inputs of the applied force/torque are given in Fig. 12, of which the values were transformed into the voltage and pulse signals for the motors. The displacement of the joints by the experiments and by the simulation are shown in Fig. 13. In the steady state, the deviation between the experimental value and the computational value is about $2.5 \%$ of the magnitude.
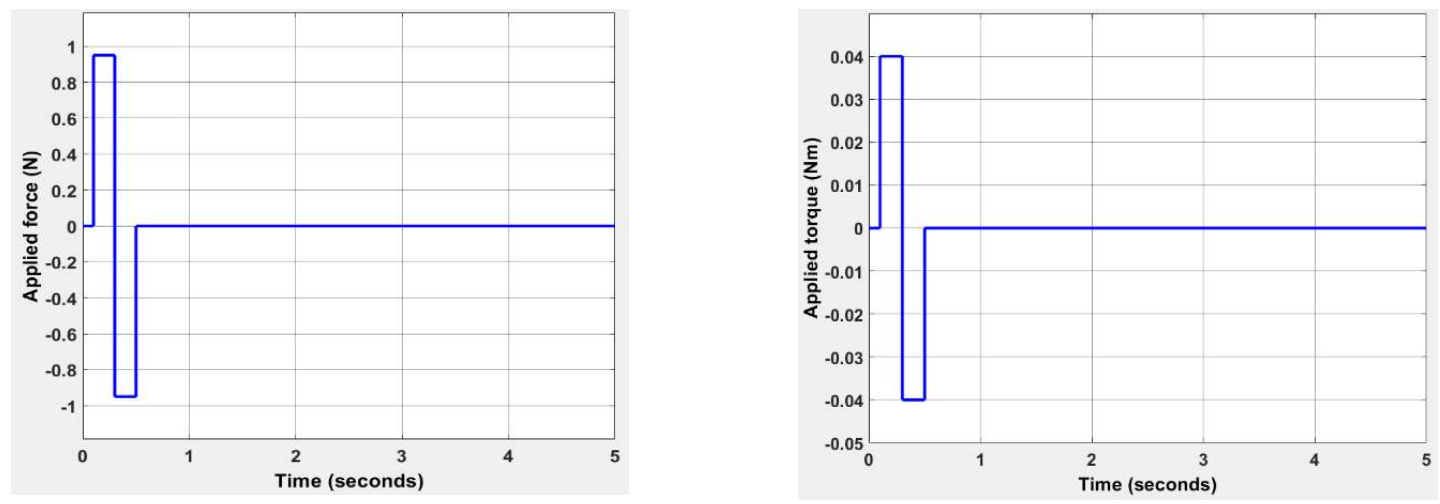

Fig. 12. The applied force/torque for the experiment

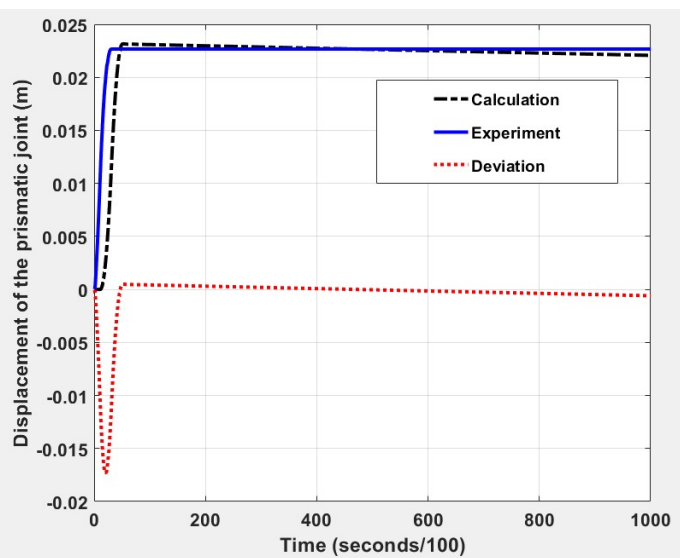

(a)

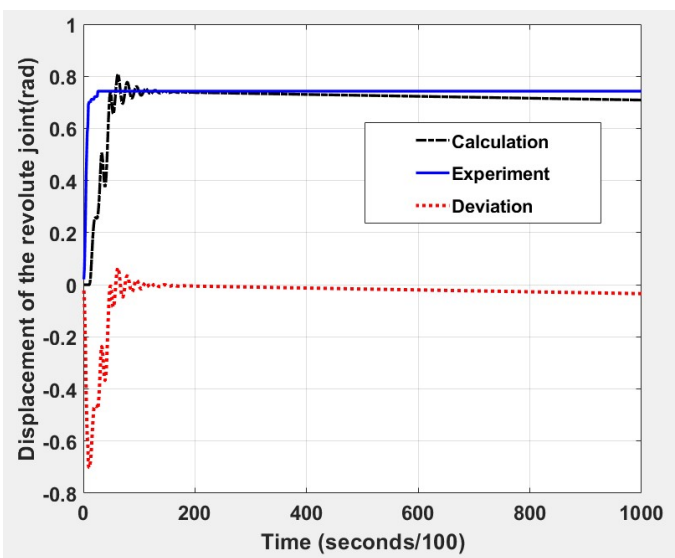

(b)

Fig. 13. The joint displacements. (a): A displacement of the prismatic joint. (b): A displacement of the revolute joint

Fig. 14 presents the flexural displacement at the second node of the first element of a link 2, and the flexural displacement at the arm tip. Fig. 15 shows the deviation between the computational value and the experimental value of both the flexural displacements mentioned in Fig.14. It can be seen that the shape and the value of the simulation curves and the experimental readings are well matching, which shows a good validation of the proposed dynamic modelling and analysis results. 

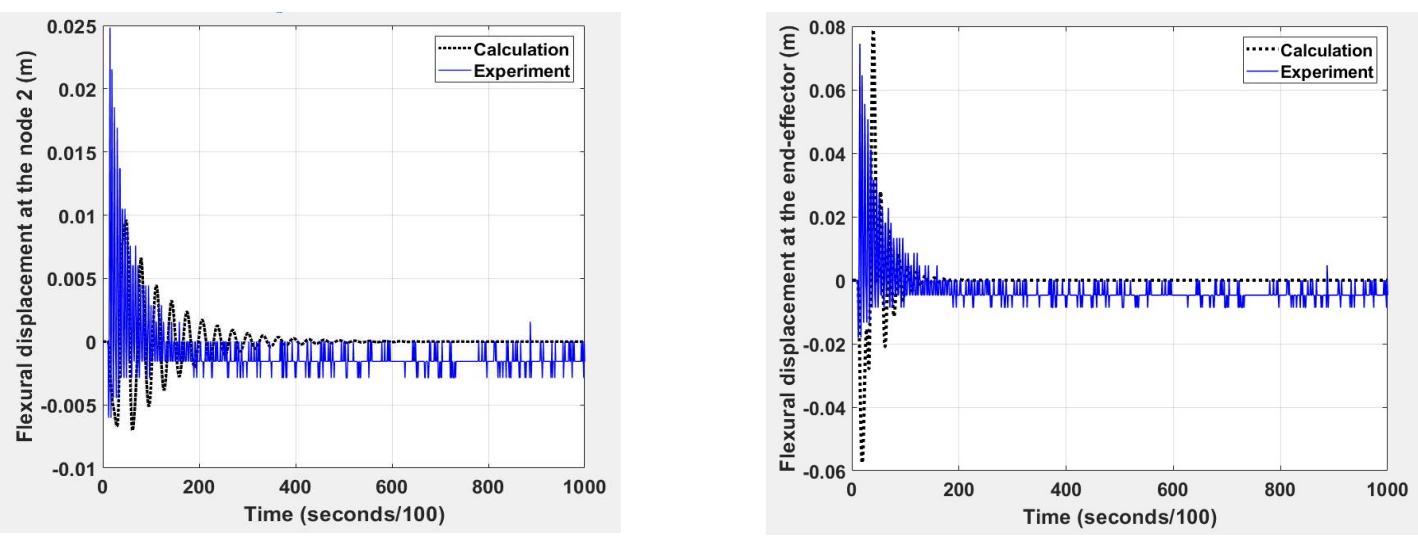

Fig. 14. The flexural displacement at the second node of the first element of a link 2 and at the arm tip.
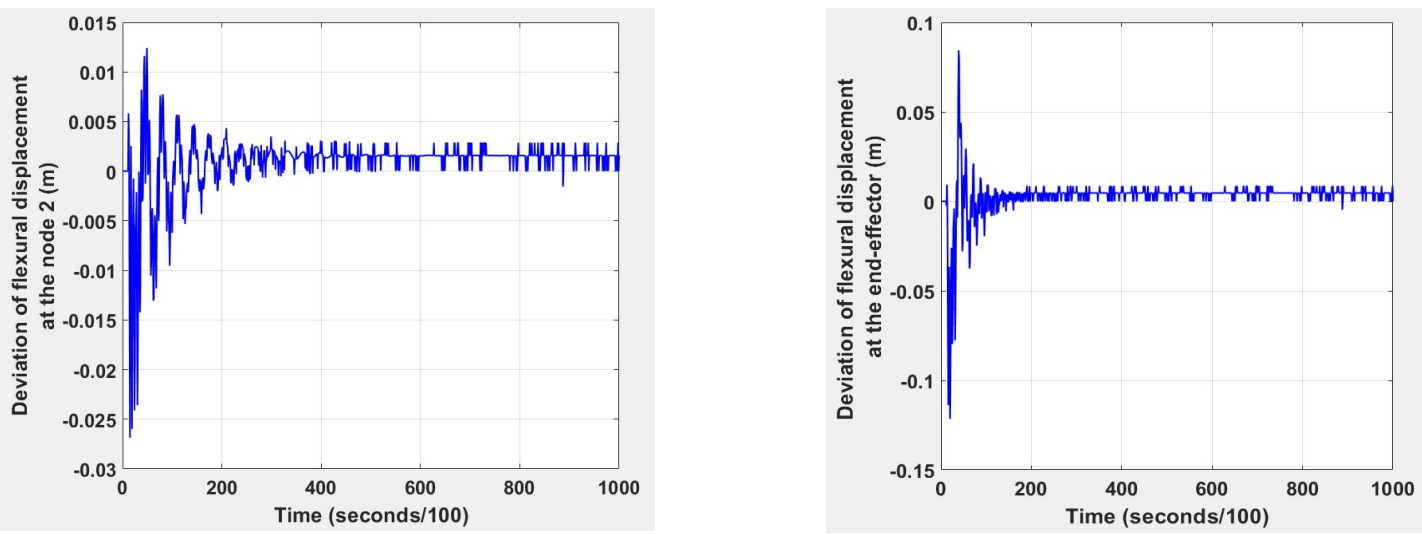

Fig. 15. A deviation between the computational value and the experimental value of both the flexural displacements

\section{Discussions and Conclusions}

There are different studies of using FEM to formulate the dynamic equations of the flexible robots. Based on the Lagrangian formulation, the dynamic equations are derived for (i) One revolute joint flexible robot (Tokhi et al., 2001; Al-Bedoor and Almusallam, 2000; and Mahto, 2014), (ii) Two link manipulator consisting of two revolute joints (Karagulle et al., 2015, 2017), (iii) One link flexible robot with one fixed prismatic joint (Al-Bedoor \& Khulief, 1997; and Pan et al., 1990), (iv) Sliding prismatic joints of a parallel robot (Wang \& Mills, 2006), (v) Multi-link robots (Usoro, 1990). Meanwhile, based on the Newton Euler method, Jonker (1990) and Naganathan \& Soni (1987) addressed the finite element formulations of the dynamic equations, in which the elemental matrices were derived with respect to the nodal degrees of freedom.

It should be noticed that, in parallel with the reviewed studies using FEM to formulate the dynamic equations for the flexible robots, there have also been researches using AMM to formulate recursively the dynamic equations for multibody systems and special flexible robotic systems. Lugris et al. (2007) approximated the elastic displacements at a node in a flexible body of a multibody system by using a linear combination of static and dynamic deformation modes with respect to modal amplitudes. Kim and Haug $(1988,1989)$ used AMM to proposed a recursive formulation for flexible multibody dynamics. By using AMM incorporated with Gibbs-Appell formulation, Korayem \& Shafei $(2013,2015)$ derived recursively the dynamic equations for some complex flexible robotic systems: the $\mathrm{N}$-viscoelastic manipulators and the $\mathrm{N}$-viscoelastic link manipulators mounted on a mobile platform. Meanwhile, Korayem et al. (2012) investigated the dynamic formulation for the mobile manipulator with elastic joints. 
It is clearly seen that most of the previous finite element methods of the dynamic modelling did emphasize on the specific configurations of a flexible robot that consists of one or two links. Although Usoro (1990), Jonker (1990) and Naganathan \& Soni (1987) developed the dynamic formulation for a multi-link robot, it is only used for the case of the robot that consists of all revolute joints. In this study, the kinematic and dynamic modelling was successfully investigated for the case of a multi-link flexible robot that consists of the revolute joints, the fixed prismatic joints and the sliding prismatic joints. The use of the multiple types of joints for a multi-link robot allows to maximise the flexibility in the design and functionality of a robot, to meet well more complex technical requirements and customer need.

In the previously proposed dynamic modelling methods, each elemental matrix is usually expressed in terms of four nodal elastic deformations of the corresponding element. Thus, the index of the last four rows and the last four columns of each elemental matrix is different from that of the other elemental matrices. The global matrices must be assembled from all elemental matrices. Assembling the global mass and stiffness matrices from total $2 \eta$ elemental mass and stiffness matrices involves a computational procedure with the time complexity of $\mathrm{O}(2 \eta)$. This because the computational procedure requires at least two computational loops with a time complexity of $\mathrm{O}(2 \eta)$ for assembling the global mass and stiffness matrices. In the dynamic modelling method of this study, such the complex procedure is not required. The global mass and stiffness matrices are effectively computed by summing all over elemental mass and stiffness matrices of the same size, respectively. The symbolic formulation is presented in a general and compact form that is ready for the symbolic programming. It is even more efficient for the cases in which a large number of the finite elements on the links is considered.

In conclusions, a new kinematic and dynamic modelling method of a multi-link planar flexible robot is successfully developed. Based on the Finite Element - Lagrangian approach, the kinematic modeling issues of the robot are generalized, taking into accounts the flexibility effects of links. For the arbitrary pair of the links, a new definition of the local coordinate system that is attached to each link is introduced. In addition, a new transformation matrix that describes the kinematics of both the motion of the joint and the deformation of the links was well addressed and discussed in details. It is shown that the proposed transformation matrix in this study is applicable to work on the dynamic modelling of any planar flexible robots. The additional contribution of this study is that a recursive symbolic formulation of the dynamic equations is newly introduced to the field of robotics. In comparison to the previously proposed methods, the time complexity of the formulation is reduced by $\mathrm{O}(2 \eta)$, where $\eta$ is the number of beam elements on the links. Furthermore, it was well demonstrated that the proposed dynamic modelling formulation can be implemented in a simplified and efficient manner, by using the symbolic computing syntaxes that are available in the symbolic and numeric computing environments such as MATLAB and Maple. Finally, the numerical examples and experiments were implemented to validate the proposed dynamic modelling method; and it clearly shows a close match about the results between the simulation and the experiment.

\section{Funding statement}

This research is funded by Vietnam National Foundation for Science and Technology Development (NAFOSTED) under the grant number 107.04-2017.09.

\section{Declaration of conflicting interests}

The authors declare that there is no conflict of interest.

\section{References}

Abbas LK, Zhou Q, Bestle D and Rui X (2017) A unified approach for treating linear multibody systems involving flexible beams. Mechanism and Machine Theory 107: 197-209. 
Abe A (2009) Trajectory planning for residual vibration suppression of a two-link rigid-flexible manipulator considering large deformation. Mechanism and Machine Theory 44(9): 1627-1639.

Alandoli EA, Sulaiman M, Rashid MZA, Shah HNM and Ismail Z (2016) A Review Study on Flexible Link Manipulators. Journal of Telecommunication, Electronic and Computer Engineering 8(2): 93-97.

Al-Bedoor BO and Almusallam AA (2000) Dynamics of flexible-link and flexible-joint manipulator carrying a payload with rotary inertia. Mechanism and Machine Theory 35(6): 785-820.

Al-Bedoor BO and Khulief YA (1997) General planar dynamics of a sliding flexible link. Journal of Sound and Vibration 206(5): 641-661.

Amirouche FML and Xie M (1993) An explicit matrix formulation of the dynamical equations for flexible multibody systems: a recursive approach. Computers \& structures 46(2): 311-321.

Asada H, Ma ZD and Tokumaru H (1990) Inverse dynamics of flexible robot arms: modeling and computation for trajectory control. Journal of dynamic systems, Measurement, and Control 112(2): 177-185.

Ata AA, Fares WF and Saadeh MY (2012) Dynamic analysis of a two-link flexible manipulator subject to different sets of conditions. Procedia Engineering 41: 1253-1260.

Augustynek K and Adamiec-Wójcik I (2012) Analysis of mechanisms with flexible beam-like links, rotary joints and assembly errors. Archive of Applied Mechanics 82(2): 283-295.

Benosman M and Le Vey G (2004) Control of flexible manipulators: A survey. Robotica 22(5): 533545.

Bolandi H and Esmaeilzadeh SM (2011) Exact Tip Trajectory Tracking Control of a Flexible Robot Arm. International Journal of Robotics and Automation 26(1): 100.

Book WJ (1984) Recursive Lagrangian dynamics of flexible manipulator arms. The International Journal of Robotics Research 3(3): 87-101.

Boyer F and Coiffet P (1996) Symbolic modeling of a flexible manipulator via assembling of its generalized Newton Euler model. Mechanism and Machine Theory 31(1): 45-56.

Boyer F and Khalil W (1998) An efficient calculation of flexible manipulator inverse dynamics. The International Journal of Robotics Research 17(3): 282-293. 
Bricout JN, Debus JC and Micheau P (1990) A finite element model for the dynamics of flexible manipulators. Mechanism and Machine Theory 25(1): 119-128.

Briot S and Khalil W (2014) Recursive and symbolic calculation of the elastodynamic model of flexible parallel robots. The International Journal of Robotics Research 33(3): 469-483.

Cannon RH and Schmitz E (1984) Initial experiments on the end-point control of a flexible one-link robot. The International Journal of Robotics Research 3(3): 62-75.

Changizi K, Shabana AA (1988) A recursive formulation for the dynamic analysis of open loop deformable multibody systems. Transactions of the ASME, Journal of Applied Mechanics 55 (3): 687-693.

Chen G, Zhang Z and Wang H (2018) A General Approach to the Large Deflection Problems of Spatial Flexible Rods Using Principal Axes Decomposition of Compliance Matrices. Journal of Mechanisms and Robotics 10(3): 031012.

Chen W (2001) Dynamic modeling of multi-link flexible robotic manipulators. Computers \& Structures 79(2): 183-195.

Choi SB, Lee HB and Thompson BS (1998) Compliant control of a two-link flexible manipulator by constraint Hamiltonian system. Mechanism and Machine Theory 33(3): 293-306.

Damaren CJ (2000) On the dynamics and control of flexible multibody systems with closed loops. The International Journal of Robotics Research 19(3): 238-253.

De Luca A and Siciliano B (1991) Closed-form dynamic model of planar multilink lightweight robots. IEEE Transactions on Systems, Man, and Cybernetics 21(4): 826-839.

Du H, Lim MK and Liew KM (1996) A nonlinear finite element model for dynamics of flexible manipulators. Mechanism and Machine Theory 31(8): 1109-1119.

Dwivedy SK and Eberhard P (2006) Dynamic analysis of flexible manipulators, a literature review. Mechanism and Machine Theory 41(7): 749-777.

Dwivedy SK and Eberhard P (2006) Dynamic analysis of flexible manipulators, a literature review. Mechanism and Machine Theory 41(7): 749-777.

Endo T and Kawasaki H (2014) Bending moment-based force control of flexible arm under gravity. Mechanism and Machine Theory 79: 217-229. 
Feliu V, Pereira E and Díaz IM (2014) Passivity-based control of single-link flexible manipulators using a linear strain feedback. Mechanism and Machine Theory 71: 191-208.

Frank LL, Darren MD and Chaouki TA (2004) Robot manipulator control: Theory and practice. Second edition. Marcel Dekker, Inc, New York, USA.

Geradin M, Cardona A (2001) Flexible Multibody Dynamics: A Finite Element Approach, John Wiley \& Sons, New York.

Goldenberg AA and Rakhsha F (1986) Feedforward control of a single-link flexible robot. Mechanism and Machine Theory 21(4): 325-335.

Green A and Sasiadek JZ (2004) Dynamics and trajectory tracking control of a two-link robot manipulator. Modal Analysis 10(10): 1415-1440.

Heidari HR, Korayem MH, Haghpanahi M and Batlle VF (2013) Optimal trajectory planning for flexible link manipulators with large deflection using a new displacements approach. Journal of Intelligent \& Robotic Systems 72(3-4):287-300.

Hewit JR, Morris JR, Sato K and Ackermann F (1997) Active force control of a flexible manipulator by distal feedback. Mechanism and machine theory 32(5): 583-596.

Jonker B (1990) A finite element dynamic analysis of flexible manipulators. The International Journal of Robotics Research 9(4): 59-74.

Ju J, Li W, Wang Y, Fan M and Yang X (2016) Two-Time Scale Virtual Sensor Design for Vibration Observation of a Translational Flexible-Link Manipulator Based on Singular Perturbation and Differential Games. Sensors 16(11): 1804.

Kalker JJ and Olsder GJ (1987) On robots with flexible links: dynamics, control and stability. Ingenieur-Archiv 57(1): 16-24.

Karagulle H, Malgaca L, Dirilmiş M, Akdağ M and Yavuz Ş (2015) Vibration control of a two-link flexible manipulator. The International journal of robotics research 23(12): 2023-2034.

Karagulle H, Malgaca L, Dirilmiş M, Akdağ M and Yavuz Ş (2017) Vibration control of a two-link flexible manipulator. Journal of Vibration and Control 23(12): 2023-2034. 
Khadem SE and Pirmohammadi AA (2003) Analytical development of dynamic equations of motion for a three-dimensional flexible link manipulator with revolute and prismatic joints. IEEE Transactions on Systems, Man, and Cybernetics, Part B (Cybernetics) 33(2): 237-249.

Khalil W, Boyer F and Morsli F (2017) General Dynamic Algorithm for Floating Base Tree Structure Robots With Flexible Joints and Links. Journal of Mechanisms and Robotics 9(3): 031003.

Khorrami F, Jain S, Tzes A (1992) Experiment results on an inner/outer loop controller for a two-link flexible manipulator. Proceedings of the IEEE International Conference on Robotics and Automation 103-747.

Kiang CT, Spowage A and Yoong CK (2015) Review of control and sensor system of flexible manipulator. Journal of Intelligent \& Robotic Systems 77(1): 187-213.

Kim HK, Choi SB and Thompson BS (2001) Compliant control of a two-link flexible manipulator featuring piezoelectric actuators. Mechanism and Machine Theory 36(3): 411-424.

Kim SS and Haug EJ (1988) A recursive formulation for flexible multibody dynamics: part I. Open loop systems. Computer Methods in Applied Mechanics and Engineering 71 (3): 293-314.

Kim SS and Haug EJ (1989) A recursive formulation for flexible multibody dynamics: part II. closed loop systems. Computer Methods in Applied Mechanics and Engineering 74 (3): 251-269.

Korayem MH and Dehkordi SF (2018) Derivation of motion equation for mobile manipulator with viscoelastic links and revolute-prismatic flexible joints via recursive Gibbs-Appell formulations. Robotics and Autonomous Systems 103: 175-198.

Korayem MH, Rahimi HN and Nikoobin A (2012) Mathematical modeling and trajectory planning of mobile manipulators with flexible links and joints. Applied Mathematical Modelling 36(7): 3229-3244.

Korayem MH and Shafei AM (2013) Application of recursive Gibbs-Appell formulation in deriving the equations of motion of $\mathrm{N}$-viscoelastic robotic manipulators in 3D space using Timoshenko beam theory. Acta Astronautica 83: 273-294.

Korayem MH and Shafei AM (2015) A New Approach for Dynamic Modeling of $n$ Viscoelastic-link Robotic Manipulators Mounted on a Mobile Base. Nonlinear Dynamics 79(4): 2767-2786. 
Korayem MH, Shafei AM and Dehkordi SF (2014) Systematic modeling of a chain of N-flexible link manipulators connected by revolute-prismatic joints using recursive Gibbs-Appell formulation. Archive of Applied Mechanics 84(2): 187-206.

Korayem MH, Shafei AM, Absalan F, Kadkhodaei B, Azimi A (2014) Kinematic and dynamic modeling of viscoelastic robotic manipulators using Timoshenko beam theory: theory and experiment. International Journal of Advanced Manufacturing Technology 71(5-8):1005-1018.

Korayem MH, Shafei AM, Shafei HR (2012) Dynamic Modeling of Nonholonomic Wheeled Mobile Manipulators with Elastic Joints using Recursive Gibbs-Appell Formulation. Scientia Iranica 19(4): 1092-1104.

Kwon DS and Book WJ (1994) A time-domain inverse dynamic tracking control of a single-link flexible manipulator. Journal of dynamic systems, measurement, and control 116(2): 193-200.

Li CJ, Sankar TS (1992) A systematic method of dynamics for flexible robot manipulators. Journal of robotic systems 9(7):861-91.

Li D, Zu JW and Goldenberg AA (1998) Dynamic modeling and mode analysis of flexible-link, flexible-joint robots. Mechanism and Machine Theory, 33(7): 1031-1044.

Lin J and Lewis FL (1994) A symbolic formulation of dynamic equations for a manipulator with rigid and flexible links. The International journal of robotics research 13(5): 454-466.

Lochan K, Roy BK and Subudhi B (2016) A review on two-link flexible manipulators. Annual Reviews in Control 42: 346-367.

Low KH and Vidyasagar M (1988) A Lagrangian formulation of the dynamic model for flexible manipulator systems. Journal of Dynamic Systems, Measurement, and Control 110(2): 175-181.

Lugris U, Naya MA, Gonzalez F, Cuadrado J (2007) Performance and application criteria of two fast formulations for flexible multibody dynamics. Mechanics Based Design of Structures and Machines 35(4): 381-404.

Luo ZH (1993) Direct strain feedback control of flexible robot arm: new theoretical and experimental results. IEEE Transactions on Automatic Control 38(11): 1610-1622.

Mahto S (2014) Shape optimization of revolute-jointed single link flexible manipulator for vibration suppression. Mechanism and Machine Theory 75: 150-160. 
Marghitu DB and Diaconescu CI (1999) Control techniques for impacting flexible systems. Archive of Applied Mechanics 69(8): 555-568.

Martins JM, Mohamed Z, Tokhi MO, Sa da Costa J, Botto MA (2003) Approaches for dynamic modeling of flexible manipulator systems. IEEE Proceeding on Control Theory and Applications 401-411.

Massoud HE, Naraghi M, Mahzoon M (2018) Experimental control of a flexible link by the method of Controlled Lagrangian. Journal of theoretical and applied vibration and acoustics 4(1): 8198.

Matsuno F, Fukushima S, Ohsawa Y, Kiyohara M and Sakawa Y (1987) Feedback control of a flexible manipulator with a parallel drive mechanism. The International journal of robotics research 6(4): 76-84.

Meghdari A and Fahimi F (2001) On the first-order decoupling of dynamical equations of motion for elastic multibody systems as applied to a two-link flexible manipulator. Multibody System Dynamics 5(1): 1-20.

Mehrez MW and El-Badawy AA (2010) Effect of the joint inertia on selection of under-actuated control algorithm for flexible-link manipulators. Mechanism and Machine Theory, 45(7): 967980 .

Naganathan G and Soni AH (1987) Coupling effects of kinematics and flexibility in manipulators. The International Journal of Robotics Research 6(1): 75-84.

Nagaraj BP, Nataraju BS, Ghosal A (1997) Dynamic of a two-link flexible system undergoing locking: mathematical modeling and comparison with experiments. Journal of Sound and Vibration 207(4): 567-589.

Pan YC, Scott RA and Ulsoy AG (1990) Dynamic modeling and simulation of flexible robots with prismatic joints. Journal of Mechanical Design 112(3): 307-314.

Płosa J and Wojciech S (2000) Dynamics of systems with changing configuration and with flexible beam-like links. Mechanism and Machine Theory, 35(11): 1515-1534.

Pratiher B and Dwivedy SK (2007) Non-linear dynamics of a flexible single link Cartesian manipulator. International Journal of Non-Linear Mechanics 42(9): 1062-1073. 
Rahimi HN and Nazemizadeh M (2014) Dynamic analysis and intelligent control techniques for flexible manipulators: a review. Advanced Robotics 28(2): 63-76.

Raouf F, Mohamad S, Maarouf S and Maamar B (2017) Distributed adaptive control strategy for flexible link manipulators. Robotica 35(7): 1562-1584.

Rone WS, Saab W and Ben-Tzvi P (2018) Design, Modeling, and Integration of a Flexible Universal Spatial Robotic Tail. Journal of Mechanisms and Robotics 10(4): 041001.

Sayahkarajy M, Mohamed Z and Mohd Faudzi AA (2016) Review of modelling and control of flexible-link manipulators. Proceedings of the Institution of Mechanical Engineers, Part I: Journal of Systems and Control Engineering 230(8): 861-873.

Shafei AM, Korayem MH (2017) Theoretical and experimental study of DLCC for flexible robotic arms in point-to-point motion. Optimal Control Applications and Methods 38(6): 963-972.

Shafei AM, Shafei HR (2018) Oblique Impact of Multi-Flexible-Link Systems. Journal of Vibration and Control 24(5):904-23.

Subudhi B and Morris AS (2002) Dynamic modelling, simulation and control of a manipulator with flexible links and joints. Robotics and Autonomous Systems 41(4): 257-270.

Tarvirdizadeh B and Yousefi-Koma A (2012) Dynamic object manipulation by a flexible robotic arm: theory and experiment. International Journal of Robotics and Automation 27(3): 263.

Theodore RJ and Ghosal A (1995) Comparison of the AMM and FEM for flexible multi-link manipulators. The International Journal of Robotics Research 14: 91-111.

Tokhi MO, Mohamed Z and Shaheed MH (2001) Dynamic characterisation of a flexible manipulator system. Robotica 19(5): 571-580.

Usoro PB, Nadira R and Mahil SS (1986) A finite element/Lagrange approach to modeling lightweight flexible manipulators. Journal of dynamic systems, measurement, and control 108(3): 198-205.

Wang D and Vidyasagar M (1992) Modeling a class of multilink manipulators with the last link flexible. IEEE transactions on robotics and automation 8(1): 33-41.

Wang PKC and Wei JD (1987) Vibrations in a moving flexible robot arm. Journal of Sound Vibration 116: 149-160. 
Wang X and Mills JK (2006) Dynamic modeling of a flexible-link planar parallel platform using a substructuring approach. Mechanism and Machine Theory, 41(6): 671-687.

Yim W, Zuang J, Singh SN (1993) Experimental two-axis vibration suppression and control of a flexible robot arm. Journal of Robotic Systems 10: 321- 343.

Yuh J and Young T (1991) Dynamic modeling of an axially moving beam in rotation: simulation and experiment. Journal of Dynamic Systems, Measurement, and Control 113(1): 34-40.

Zhang DG (2009) Recursive Lagrangian dynamic modeling and simulation of multi-link spatial flexible manipulator arms. Applied Mathematics and Mechanics 30(10): 1283-1294.

Zhang Y, Liu J and He W (2016) Vibration control for a nonlinear three-dimensional flexible manipulator trajectory tracking. International Journal of Control 89(8): 1641-1663.

\section{APPENDIX}

\section{Kinematics of nine configurations of the general two link flexible robot that consists of three types of joints}

\section{Schematic diagrams of the mechanisms of the robot}

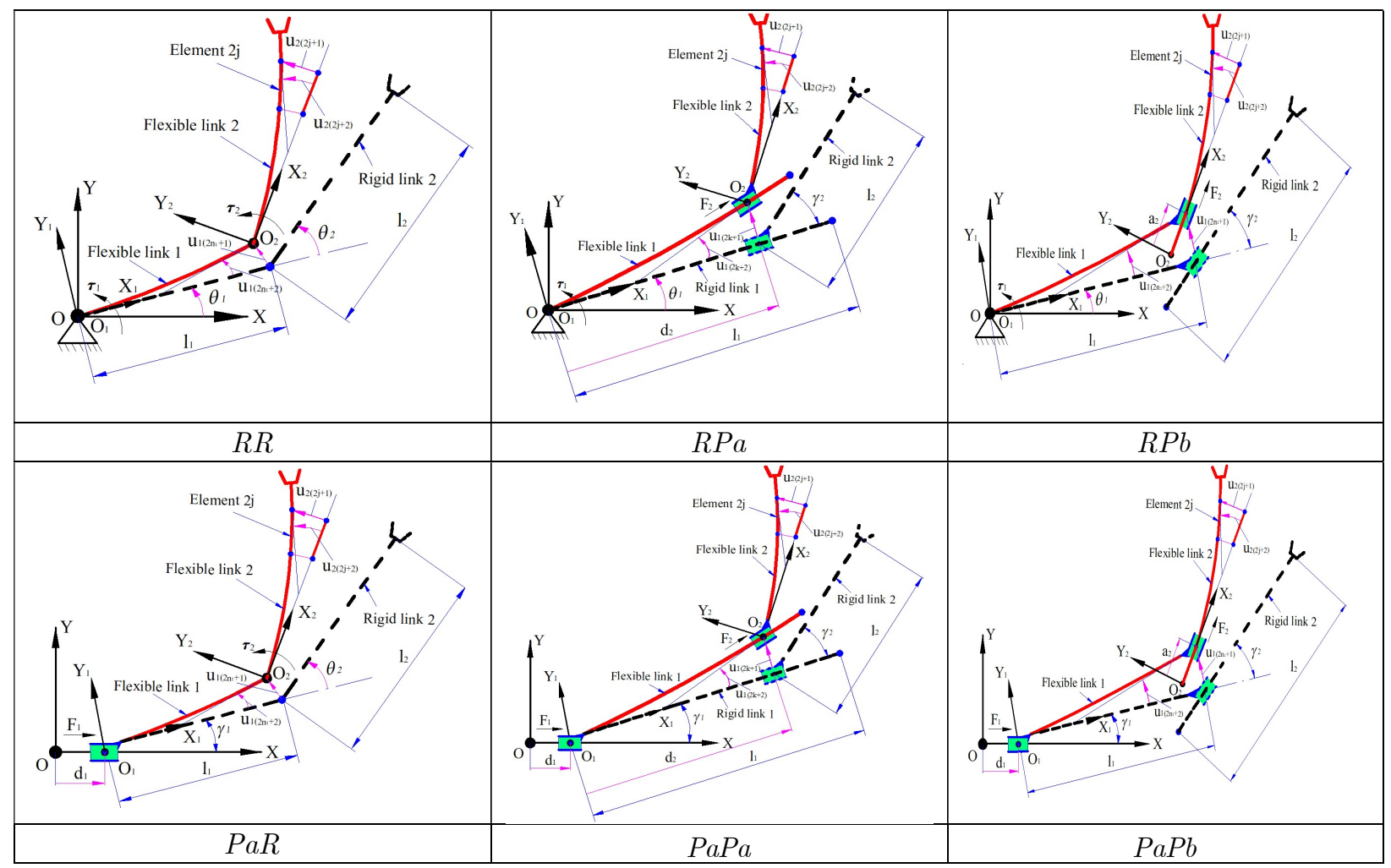




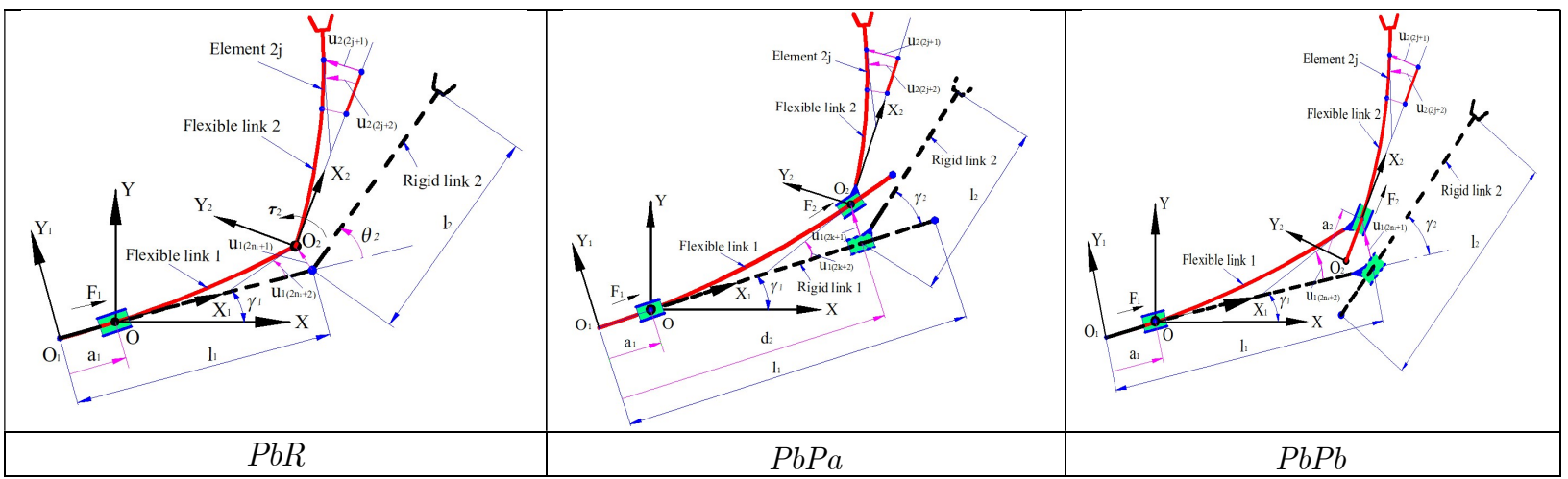

\section{The transformation matrices of the robot}

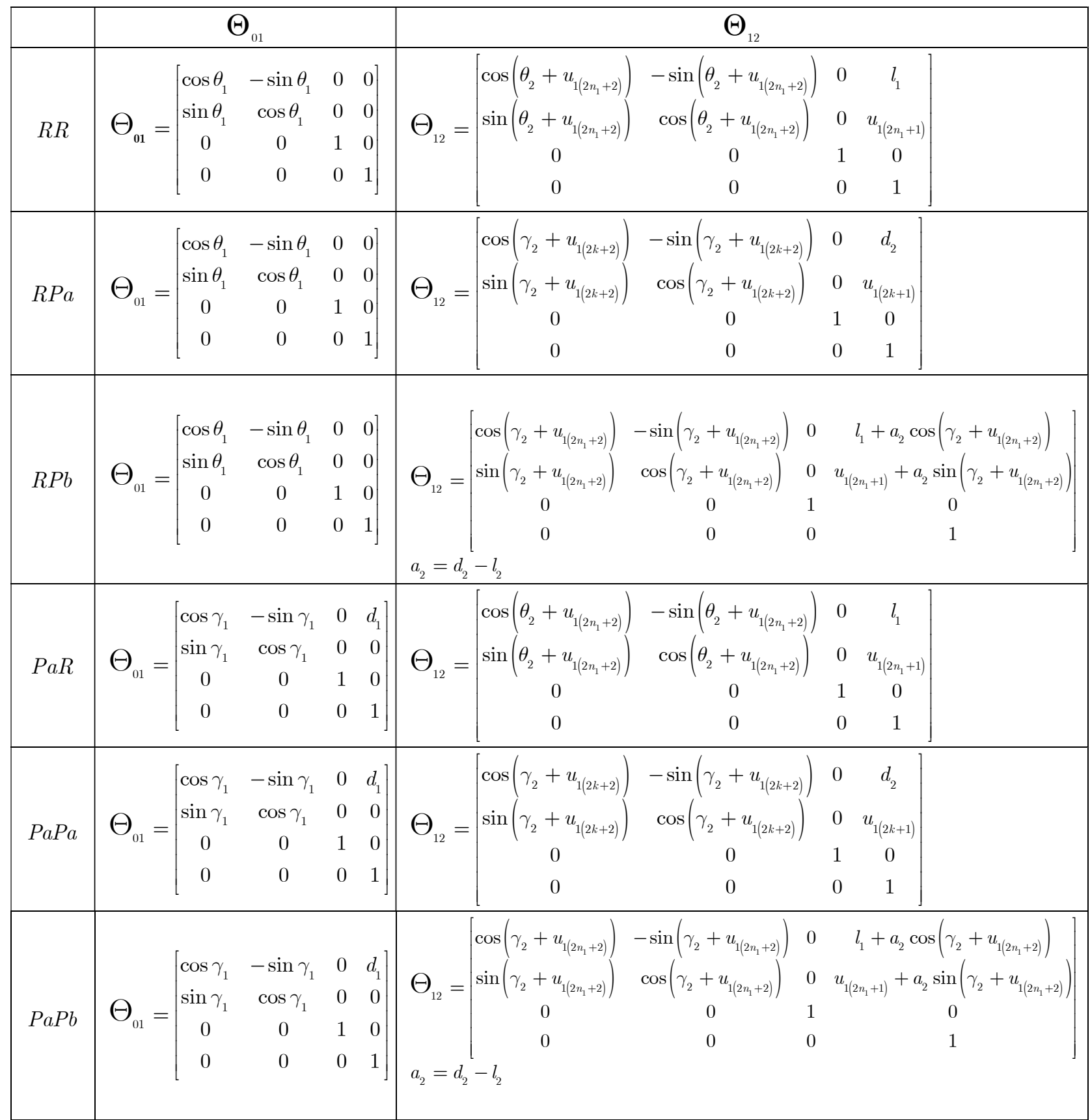




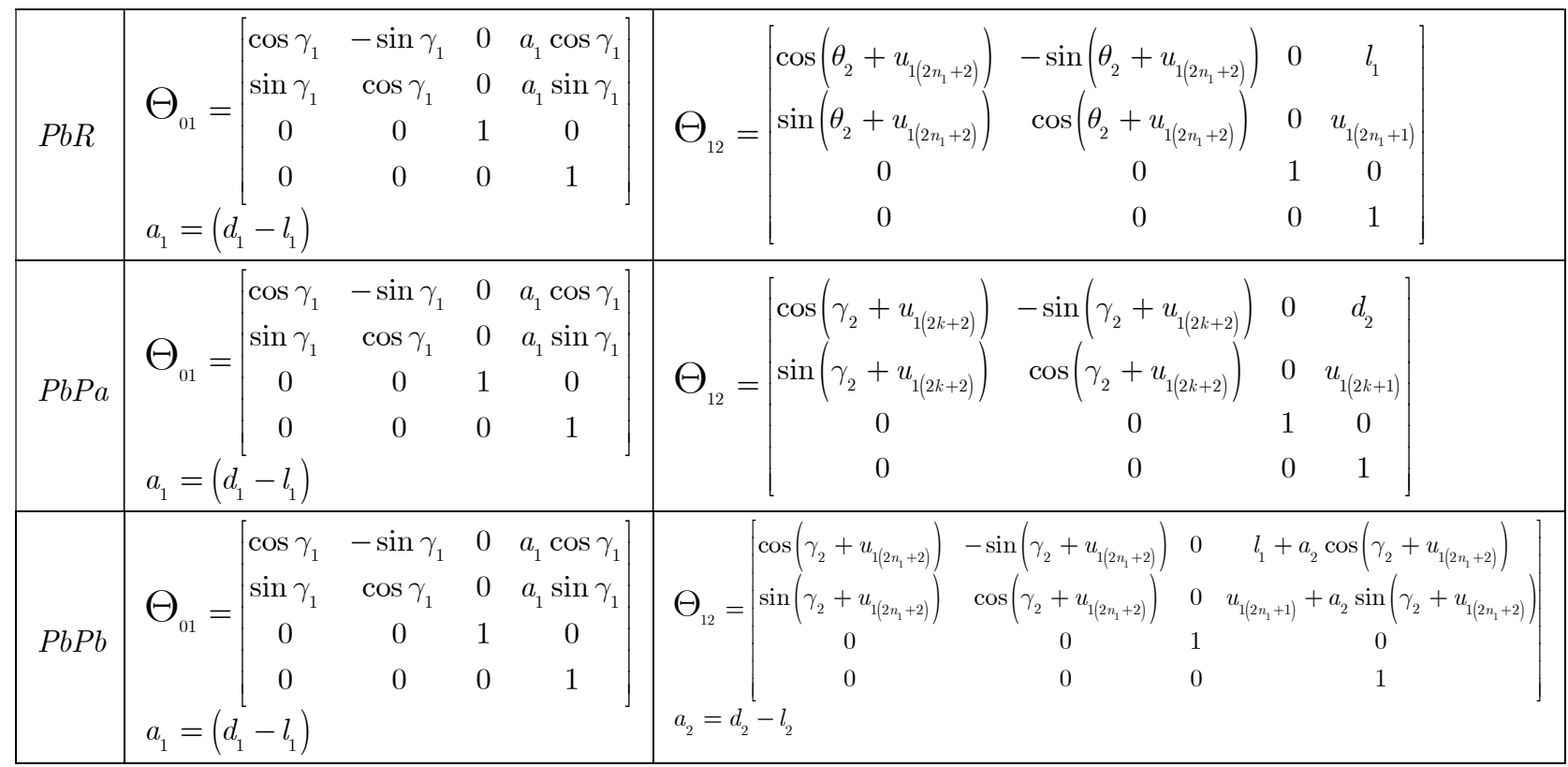

\section{The position of the distal end of the first link of a robot}

In the following table, the position of the endpoint of the first link for both the flexible robot and the rigid robot is determined. It is shown that if the flexible deformation is neglected, the kinematics of the first link of both the robots will be the same.

\begin{tabular}{|c|c|c|}
\hline & Rigid robot & Flexible robot \\
\hline $\begin{array}{l}R R \\
R P a \\
R P b\end{array}$ & $\mathbf{r}_{01}=\left[\begin{array}{l}L_{1} \cos \theta_{1} \\
L_{1} \sin \theta_{1}\end{array}\right]$ & $\mathbf{r}_{01}=\left[\begin{array}{ll}L_{1} \cos \theta_{1}-u_{1\left(2 n_{1}+1\right)} & \sin \theta_{1} \\
L_{1} \sin \theta_{1}+u_{1\left(2 n_{1}+1\right)} & \cos \theta_{1}\end{array}\right]$ \\
\hline $\begin{array}{l}P a R ; \\
P a P a ; \\
P a P b\end{array}$ & $\mathbf{r}_{01}=\left[\begin{array}{c}L_{1} \cos \gamma_{1}+d_{1} \\
L_{1} \sin \gamma_{1}\end{array}\right]$ & $\mathbf{r}_{01}=\left[\begin{array}{c}L_{1} \cos \gamma_{1}+d_{1}-u_{1\left(2 n_{1}+1\right)} \sin \gamma_{1} \\
L_{1} \sin \gamma_{1}+u_{1\left(2 n_{1}+1\right)} \cos \gamma_{1}\end{array}\right]$ \\
\hline $\begin{array}{l}P b R ; \\
P b P a ; \\
P b P b\end{array}$ & $\mathbf{r}_{01}=\left[\begin{array}{l}d_{1} \cos \gamma_{1} \\
d_{1} \sin \gamma_{1}\end{array}\right]$ & $\mathbf{r}_{01}=\left[\begin{array}{ll}d_{1} \cos \gamma_{1}-u_{1\left(2 n_{1}+1\right)} & \sin \gamma_{1} \\
d_{1} \sin \gamma_{1}+u_{1\left(2 n_{1}+1\right)} & \cos \gamma_{1}\end{array}\right]$ \\
\hline
\end{tabular}

\section{The position of the end-effector of a robot}

\begin{tabular}{|c|c|c|}
\hline & Rigid Robot & Flexible Robot \\
\hline$R R$ & $\mathbf{r}_{0 E}=\left[\begin{array}{l}L_{1} \cos \theta_{1}+L_{2} \cos \left(\theta_{1}+\theta_{2}\right) \\
L_{1} \sin \theta_{1}+L_{2} \sin \left(\theta_{1}+\theta_{2}\right)\end{array}\right]$ & $\mathbf{r}_{0 E}=\left|\begin{array}{l}L_{1} \cos \theta_{1}-u_{1\left(2 n_{1}+1\right)} \sin \theta_{1}+L_{2} \cos \left(\theta_{1}+\theta_{2}+u_{1\left(2 n_{1}+2\right)}\right) \\
-u_{2\left(2 n_{2}+1\right)} \sin \left(\theta_{1}+\theta_{2}+u_{1\left(2 n_{1}+2\right)}\right) \\
L_{1} \sin \theta_{1}+u_{1\left(2 n_{1}+1\right)} \cos \theta_{1}+L_{2} \sin \left(\theta_{1}+\theta_{2}+u_{1\left(2 n_{1}+2\right)}\right) \\
+u_{2\left(2 n_{2}+1\right)} \cos \left(\theta_{1}+\theta_{2}+u_{1\left(2 n_{1}+2\right)}\right)\end{array}\right|$ \\
\hline
\end{tabular}




\begin{tabular}{|c|c|c|c|c|}
\hline$R P a$ & $\mathbf{r}_{0 E}=$ & {$\left[\begin{array}{l}d_{2} \cos \theta_{1}+L_{2} \cos \left(\theta_{1}+\gamma_{2}\right) \\
d_{2} \sin \theta_{1}+L_{2} \sin \left(\theta_{1}+\gamma_{2}\right)\end{array}\right]$} & $\mathbf{r}_{0 E}=$ & $\left.\mid \begin{array}{l}d_{2} \cos \theta_{1}-u_{1(2 k+1)} \sin \theta_{1}+L_{2} \cos \left(\theta_{1}+\gamma_{2}+u_{1(2 k+2)}\right) \\
-u_{2\left(2 n_{2}+1\right)} \sin \left(\theta_{1}+\gamma_{2}+u_{1(2 k+2)}\right) \\
d_{2} \sin \theta_{1}+u_{1(2 k+1)} \cos \theta_{1}+L_{2} \sin \left(\theta_{1}+\gamma_{2}+u_{1(2 k+2)}\right) \\
+u_{2\left(2 n_{2}+1\right)} \sin \left(\theta_{1}+\gamma_{2}+u_{1(2 k+2)}\right)\end{array}\right]$ \\
\hline$R P b$ & $\mathbf{r}_{0 E}=$ & {$\left[\begin{array}{l}L_{1} \cos \theta_{1}+d_{2} \cos \left(\theta_{1}+\gamma_{2}\right) \\
L_{1} \sin \theta_{1}+d_{2} \sin \left(\theta_{1}+\gamma_{2}\right)\end{array}\right]$} & $\mathbf{r}_{0 E}=$ & {$\left[\begin{array}{l}L_{1} \cos \theta_{1}-u_{1\left(2 n_{1}+1\right)} \sin \theta_{1}+d_{2} \cos \left(\theta_{1}+\gamma_{2}+u_{1\left(2 n_{1}+2\right)}\right) \\
-u_{2\left(2 n_{2}+1\right)} \sin \left(\theta_{1}+\gamma_{2}+u_{1\left(2 n_{1}+2\right)}\right) \\
L_{1} \sin \theta_{1}+u_{1\left(2 n_{1}+1\right)} \cos \theta_{1}+d_{2} \sin \left(\theta_{1}+\gamma_{2}+u_{1\left(2 n_{1}+2\right)}\right) \\
+u_{2\left(2 n_{2}+1\right)} \cos \left(\theta_{1}+\gamma_{2}+u_{1\left(2 n_{1}+2\right)}\right)\end{array}\right]$} \\
\hline$P a R$ & $\mathbf{r}_{0 E}=$ & {$\left[\begin{array}{c}d_{1}+L_{1} \cos \gamma_{1} \\
+L_{2} \cos \left(\gamma_{1}+\theta_{2}\right) \\
L_{1} \sin \gamma_{1}+L_{2} \sin \left(\gamma_{1}+\theta_{2}\right)\end{array}\right]$} & $\mathbf{r}_{0 E}=$ & $\begin{array}{l}d_{1}+L_{1} \cos \gamma_{1}-u_{1\left(2 n_{1}+1\right)} \sin \gamma_{1}+L_{2} \cos \left(\gamma_{1}+\theta_{2}+u_{1\left(2 n_{1}+2\right)}\right) \\
-u_{2\left(2 n_{2}+1\right)} \sin \left(\gamma_{1}+\theta_{2}+u_{1\left(2 n_{1}+2\right)}\right) \\
\quad L_{1} \sin \gamma_{1}+u_{1\left(2 n_{1}+1\right)} \cos \gamma_{1}+L_{2} \sin \left(\gamma_{1}+\theta_{2}+u_{1\left(2 n_{1}+2\right)}\right) \\
\quad+u_{2\left(2 n_{2}+1\right)} \cos \left(\gamma_{1}+\theta_{2}+u_{1\left(2 n_{1}+2\right)}\right)\end{array}$ \\
\hline $\mathrm{PaPa}$ & $\mathbf{r}_{0 E}=$ & {$\left[\begin{array}{c}d_{1}+d_{2} \cos \gamma_{1} \\
+L_{2} \cos \left(\gamma_{1}+\gamma_{2}\right) \\
d_{2} \sin \gamma_{1}+L_{2} \sin \left(\gamma_{1}+\gamma_{2}\right)\end{array}\right]$} & $\mathbf{r}_{0 E}=$ & $\begin{array}{l}d_{1}+d_{2} \cos \gamma_{1}-u_{1(2 k+1)} \sin \gamma_{1}+L_{2} \cos \left(\gamma_{1}+\gamma_{2}+u_{1(2 k+2)}\right) \\
-u_{2\left(2 n_{2}+1\right)} \sin \left(\gamma_{1}+\gamma_{2}+u_{1(2 k+2)}\right) \\
\quad d_{2} \sin \gamma_{1}+u_{1(2 k+1)} \cos \gamma_{1}+L_{2} \sin \left(\gamma_{1}+\gamma_{2}+u_{1(2 k+2)}\right) \\
\quad+u_{2\left(2 n_{2}+1\right)} \cos \left(\gamma_{1}+\gamma_{2}+u_{1(2 k+2)}\right)\end{array}$ \\
\hline $\mathrm{PaPb}$ & $\mathbf{r}_{0 E}=$ & {$\left[\begin{array}{c}d_{1}+L_{1} \cos \gamma_{1} \\
+d_{2} \cos \left(\gamma_{1}+\gamma_{2}\right) \\
L_{1} \sin \gamma_{1}+d_{2} \sin \left(\gamma_{1}+\gamma_{2}\right)\end{array}\right]$} & $\mathbf{r}_{0 E}=$ & $\begin{array}{l}d_{1}+L_{1} \cos \gamma_{1}-u_{1\left(2 n_{1}+1\right)} \sin \gamma_{1}+d_{2} \cos \left(\gamma_{1}+\gamma_{2}+u_{1\left(2 n_{1}+2\right)}\right) \\
-u_{2\left(2 n_{2}+1\right)} \sin \left(\gamma_{1}+\gamma_{2}+u_{1\left(2 n_{1}+2\right)}\right) \\
\quad L_{1} \sin \gamma_{1}+u_{1\left(2 n_{1}+1\right)} \cos \gamma_{1}+d_{2} \sin \left(\gamma_{1}+\gamma_{2}+u_{1\left(2 n_{1}+2\right)}\right) \\
\quad+u_{2\left(2 n_{2}+1\right)} \cos \left(\gamma_{1}+\gamma_{2}+u_{1\left(2 n_{1}+2\right)}\right)\end{array}$ \\
\hline$P b R$ & $\mathbf{r}_{0 E}=$ & {$\left[\begin{array}{l}d_{1} \cos \gamma_{1}+L_{2} \cos \left(\gamma_{1}+\theta_{2}\right) \\
d_{1} \sin \gamma_{1}+L_{2} \sin \left(\gamma_{1}+\theta_{2}\right)\end{array}\right]$} & $\mathbf{r}_{0 E}=$ & $\begin{array}{l}d_{1} \cos \gamma_{1}-u_{1\left(2 n_{1}+1\right)} \sin \gamma_{1}+L_{2} \cos \left(\gamma_{1}+\theta_{2}+u_{1\left(2 n_{1}+2\right)}\right) \\
-u_{2\left(2 n_{2}+1\right)} \sin \left(\gamma_{1}+\theta_{2}+u_{1\left(2 n_{1}+2\right)}\right) \\
d_{1} \sin \gamma_{1}+u_{1\left(2 n_{1}+1\right)} \cos \gamma_{1}+L_{2} \sin \left(\gamma_{1}+\theta_{2}+u_{1\left(2 n_{1}+2\right)}\right) \\
+u_{2\left(2 n_{2}+1\right)} \cos \left(\gamma_{1}+\theta_{2}+u_{1\left(2 n_{1}+2\right)}\right)\end{array}$ \\
\hline
\end{tabular}




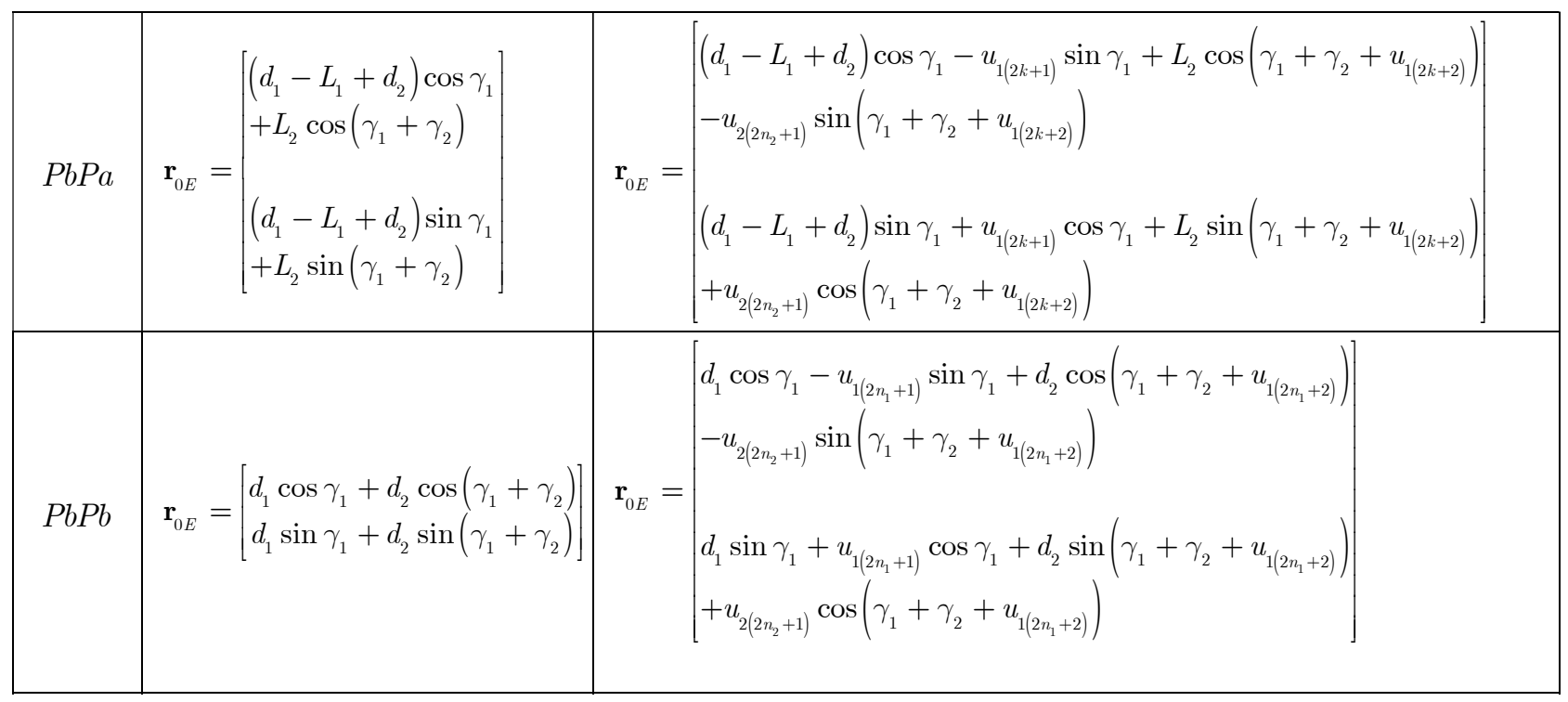

